\title{
The compositional evolution of C/2012 S1 (ISON) from ground-based high-resolution infrared spectroscopy as part of a worldwide observing campaign
}

\author{
N. Dello Russo a,*, R.J. Vervack Jr. ${ }^{a}$, H. Kawakita ${ }^{b}$, A. Cochran ${ }^{c}$, A.J. McKay ${ }^{c}$, W.M. Harris ${ }^{\text {d }}$, H.A. Weaver ${ }^{a}$, \\ C.M. Lisse ${ }^{a}$, M.A. DiSanti ${ }^{\text {e }}$, H. Kobayashi ${ }^{b}$, N. Biver ${ }^{f}$, D. Bockelée-Morvan ${ }^{f}$, J. Crovisier ${ }^{\text {f }}$, C. Opitom ${ }^{g}$, \\ E. Jehin ${ }^{g}$
}

${ }^{a}$ The Johns Hopkins University Applied Physics Laboratory, Laurel, MD 20723-6099, USA

${ }^{\mathrm{b}}$ Koyama Astronomical Observatory, Kyoto Sangyo University Motoyama, Kamigamo, Kita-ku, Kyoto 603-8555, Japan

${ }^{\mathrm{C}}$ McDonald Observatory, 2515 Speedway, Stop C1402, Austin, TX 78712, USA

${ }^{\mathrm{d}}$ Lunar and Planetary Laboratory, University of Arizona, Tucson, AZ 85721, USA

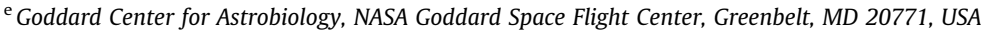

${ }^{\mathrm{f}}$ LESIA, Observatoire de Paris, 5 Place Jules Janssen, 92195 Meudon, France

${ }^{\mathrm{g}}$ F.R.S.-FNRS, Institut d'Astrophysique et de Géophysique, Université de Liège, Allée du 6 août 17, 4000 Liège, Belgium

\section{A R T I C L E I N F O}

\section{Article history:}

Received 8 May 2015

Revised 27 October 2015

Accepted 18 November 2015

Available online 11 December 2015

\section{Keywords:}

Comets

Infrared observations

Spectroscopy

\begin{abstract}
A B S T R A C T
Volatile production rates, relative abundances, rotational temperatures, and spatial distributions in the coma were measured in C/2012 S1 (ISON) using long-slit high-dispersion $\left(\lambda / \Delta \lambda \sim 2.5 \times 10^{4}\right)$ infrared spectroscopy as part of a worldwide observing campaign. Spectra were obtained on UT 2013 October 26 and 28 with NIRSPEC at the W.M. Keck Observatory, and UT 2013 November 19 and 20 with CSHELL at the NASA IRTF. $\mathrm{H}_{2} \mathrm{O}$ was detected on all dates, with production rates increasing markedly from $(8.7 \pm 1.5) \times 10^{27}$ molecules s ${ }^{-1}$ on October $26\left(R_{h}=1.12 \mathrm{AU}\right)$ to $(3.7 \pm 0.4) \times 10^{29}$ molecules s $^{-1}$ on November $20\left(R_{h}=0.43 \mathrm{AU}\right)$. Short-term variability of $\mathrm{H}_{2} \mathrm{O}$ production is also seen as observations on November 19 show an increase in $\mathrm{H}_{2} \mathrm{O}$ production rate of nearly a factor of two over a period of about 6 h. $\mathrm{C}_{2} \mathrm{H}_{6}, \mathrm{CH}_{3} \mathrm{OH}$ and $\mathrm{CH}_{4}$ abundances in ISON are slightly depleted relative to $\mathrm{H}_{2} \mathrm{O}$ when compared to mean values for comets measured at infrared wavelengths. On the November dates, $\mathrm{C}_{2} \mathrm{H}_{2}, \mathrm{HCN}$ and OCS abundances relative to $\mathrm{H}_{2} \mathrm{O}$ appear to be within the range of mean values, whereas $\mathrm{H}_{2} \mathrm{CO}$ and $\mathrm{NH}_{3}$ were significantly enhanced. There is evidence that the abundances with respect to $\mathrm{H}_{2} \mathrm{O}$ increased for some species but not others between October $28\left(R_{h}=1.07 \mathrm{AU}\right)$ and November $19\left(R_{h}=0.46 \mathrm{AU}\right)$. The high mixing ratios of $\mathrm{H}_{2} \mathrm{CO} / \mathrm{CH}_{3} \mathrm{OH}$ and $\mathrm{C}_{2} \mathrm{H}_{2} / \mathrm{C}_{2} \mathrm{H}_{6}$ on November 19 , and changes in the mixing ratios of some species with respect to $\mathrm{H}_{2} \mathrm{O}$ between October 28 to November 19, indicates compositional changes that may be the result of a transition from sampling radiation-processed outer layers in this dynamically new comet to sampling more pristine natal material as the outer processed layer was increasingly eroded and the thermal wave propagated into the nucleus as the comet approached perihelion for the first time. On November 19 and 20, the spatial distribution for dust appears asymmetric and enhanced in the antisolar direction, whereas spatial distributions for volatiles (excepting $\mathrm{CN}$ ) appear symmetric with their peaks slightly offset in the sunward direction compared to the dust. Spatial distributions for $\mathrm{H}_{2} \mathrm{O}, \mathrm{HCN}, \mathrm{C}_{2} \mathrm{H}_{6}, \mathrm{C}_{2} \mathrm{H}_{2}$, and $\mathrm{H}_{2} \mathrm{CO}$ on November 19 show no definitive evidence for significant contributions from extended sources; however, broader spatial distributions for $\mathrm{NH}_{3}$ and OCS may be consistent with extended sources for these species. Abundances of $\mathrm{HCN}$ and $\mathrm{C}_{2} \mathrm{H}_{2}$ on November 19 and 20 are insufficient to account for reported abundances of $\mathrm{CN}$ and $\mathrm{C}_{2}$ in ISON near this time. Differences in $\mathrm{HCN}$ and $\mathrm{CN}$ spatial distributions are also consistent with $\mathrm{HCN}$ as only a minor source of $\mathrm{CN}$ in ISON on November 19 as the spatial distribution of $\mathrm{CN}$ in the coma suggests a dominant distributed source that is correlated with dust and not volatile release. The spatial distributions for $\mathrm{NH}_{3}$ and $\mathrm{NH}_{2}$ are similar, suggesting that $\mathrm{NH}_{3}$ is the primary source of $\mathrm{NH}_{2}$ with no evidence of a significant dust source of $\mathrm{NH}_{2}$; however, the higher production rates derived for $\mathrm{NH}_{3} \mathrm{Com}$ pared to $\mathrm{NH}_{2}$ on November 19 and 20 remain unexplained. This suggests a more complete analysis that treats $\mathrm{NH}_{2}$ as a distributed source and accounts for its emission mechanism is needed for future work.
\end{abstract}

(c) 2015 Elsevier Inc. All rights reserved.

\footnotetext{
* Corresponding author.
} 


\section{Background}

C/2012 S1 (ISON) (hereafter ISON) was discovered on September 21, 2012, by Vitali Nevski and Artyom Novichonok at the 0.4$\mathrm{m}$ reflector of the International Scientific Optical Network (ISON) when it was at a distance of 6.3 AU from the Sun. Shortly thereafter, orbital determinations indicated that the comet was likely dynamically new and would make a perihelion passage extremely close to the Sun (0.012 AU) on 28 November 2013. The detection of ISON at such a large heliocentric distance suggested that it could be large and might become quite bright. In addition, there was a long period of favorable observing conditions leading up to perihelion, followed by a close post-perihelion approach to Earth (0.42 AU) in December 2013. Because sungrazing comets are usually small and discovered near perihelion, the long-term availability of ISON for observations presented a unique opportunity to study the evolution of a dynamically new sungrazing comet for many months prior to perihelion, before it experienced extreme heating for the first time, and then potentially through and past perihelion.

These unique circumstances led NASA to organize the Comet ISON Observing Campaign ( $\mathrm{CIOC}$ ) to coordinate observations of ISON from many different facilities covering a broad spectral range. Based on orbital and brightness considerations, the optimal time for infrared spectroscopic observations spanned from October 2013 to January 2014. Planning of infrared spectroscopic observations was especially challenging because the brightness evolution and the survivability of ISON as it approached perihelion were highly uncertain at the time when observing proposals were solicited (March and April 2013). Additionally, ISON had limited dark-time availability during optimal observing times; therefore, daytime observations were required for much of the prime period when the comet was close to the Sun. Accounting for these circumstances, NASA, the CIOC, the W.M. Keck Observatory, and the NASA Infrared Telescope Facility (IRTF) worked together to put aside blocks of time, enabling an efficient scientific study of ISON.

We obtained a total of sixteen blocks of time to observe ISON at Keck and IRTF with eight blocks each pre- and post-perihelion. The light-gathering power of Keck enabled fruitful observations within 3-h blocks of dark-time when the comet was farther from the Sun. The daytime observing capability of IRTF allowed observations of ISON when it was brighter and closer to the Sun. Four scheduled pre-perihelion blocks were completely lost (November $11,12,14$, and 21) and two others were partially lost (October 26 and November 20) due to poor weather. All post-perihelion nights were lost with the complete disruption of the nucleus of ISON near perihelion. We thus report here $\mathrm{H}_{2} \mathrm{O}$ rotational temperatures, volatile production rates, and spatial distributions in the coma of ISON on four pre-perihelion dates. In Section 2 we discuss how observations were obtained and our methodology for analyzing these data. In Section 3 we discuss our results in detail, including the evolution of observed $\mathrm{H}_{2} \mathrm{O}$ production rates, the derived rotational temperatures of $\mathrm{H}_{2} \mathrm{O}$ in the coma, production rates and relative abundances of $\mathrm{C}_{2} \mathrm{H}_{6}, \mathrm{CH}_{3} \mathrm{OH}, \mathrm{CH}_{4}, \mathrm{C}_{2} \mathrm{H}_{2}, \mathrm{HCN}, \mathrm{H}_{2} \mathrm{CO}, \mathrm{NH}_{3}$, $\mathrm{NH}_{2}$ and OCS with respect to $\mathrm{H}_{2} \mathrm{O}$, and how the volatile chemistry in ISON evolved with time and how it compares with other comets. Because we determined spatial distributions for $\mathrm{H}_{2} \mathrm{O}$, $\mathrm{C}_{2} \mathrm{H}_{6}, \mathrm{C}_{2} \mathrm{H}_{2}, \mathrm{HCN}, \mathrm{H}_{2} \mathrm{CO}, \mathrm{NH}_{3}, \mathrm{NH}_{2}$, OCS and $\mathrm{CN}$ in the coma, we also discuss the potential sources of these volatiles in ISON. In particular, we compare abundances and spatial distributions for parent volatile species $\mathrm{HCN}$ and $\mathrm{NH}_{3}$ to potential corresponding daughter species $\mathrm{CN}$ and $\mathrm{NH}_{2}$, thus providing information on the possible sources for these daughter species in ISON. Our overall findings are summarized in Section 4.

\section{Observations \& data analysis}

Observations on UT October 26 and 28 (all presented dates and times hereafter are UT unless otherwise noted) were obtained with the NIRSPEC instrument (McLean et al., 1998) at the 10-m W.M. Keck Observatory on Mauna Kea, Hawaii, when ISON was predicted to be sufficiently productive and still available at night. Observations on November 19 and 20 were obtained with the CSHELL spectrometer (Greene et al., 1993) at the 3-m NASA IRTF on Mauna Kea, Hawaii, during daytime when the Sun-observer-ISON angle was between $25^{\circ}$ and $28^{\circ}$. Although challenging, comet observations with IRTF/CSHELL have been successfully performed in the past under similar conditions (e.g. Dello Russo et al., 2009a; DiSanti et al., 2009). The observing circumstances for all ISON observations are summarized in Table 1.

For the ISON observations with NIRSPEC we used a $24^{\prime \prime} \times 0^{\prime \prime} .432$ slit, resulting in a spectral resolving power $(\lambda \mid \Delta \lambda) \sim 28,000$. At each grating setting, comet spectra were acquired using a sequence of four scans with an integration time of $1 \mathrm{~min}$ on-source per scan (4 min for the complete sequence). During a sequence of scans the telescope was nodded $12^{\prime \prime}$ between $A$ and $B$ positions in an ABBA pattern, keeping the comet on-slit for all integrations with the midpoint of the slit approximately equidistant from the $A$ and $\mathrm{B}$ positions. The slit was set in position angle mode at $282^{\circ}$ on October 26. Because of the observed faintness of ISON on October 26 and for operational simplicity, the slit was instead set in the nominal, physically fixed position on NIRSPEC on October 28 , allowing the slit to rotate on the sky during those observations. At each grating setting spectra of infrared standard stars were taken: HR 4227 on October 26 and HR 3134 on October 28. The widest NIRSPEC slit is only five pixels $\left(0^{\prime \prime} .74\right)$ wide, so corrections for slit losses were included in the NIRSPEC flux calibration analysis. The average seeing (FWHM) on both dates was about $0^{\prime \prime} .5$.

CSHELL has a $256 \times 256$-pixel InSb array detector with a pixel size of $0^{\prime \prime} .2 \times 0^{\prime \prime} .2$ resulting in $\lambda / \Delta \lambda \sim 25,000$ for the 5-pixel $\left(1^{\prime \prime}\right)$ wide slit and providing spatial coverage along the $30^{\prime \prime}$ length of the slit. During a sequence of scans the telescope was nodded $15^{\prime \prime}$ between $A$ and $B$ positions in an ABBA pattern, keeping the comet on-slit for all integrations with the midpoint of the slit approximately equidistant from the A and B positions. The CSHELL slit was oriented east-west during observations on November 19 and 20 when the position angle of the extended Sun-comet radius vector was $286^{\circ}$; therefore, the Sun position in the plane of the slit was at an angle of $16^{\circ}$ south of east. The Sun-comet-observer angle on these dates ranged over about $91-94^{\circ}$, so the Sun was almost in the plane of the slit from the standpoint of the observer. All CSHELL observations of ISON occurred during daylight when tracking non-sidereal objects is complicated because the autoguiding capability of the telescope cannot be used and atmospheric seeing is generally poor and variable. Images of the comet were therefore taken before and after each sequence of scans to assess cometary drift and update telescope tracking rates as needed. To minimize drift within a sequence of scans, integration times on the comet were limited to one hundred and sixty seconds per scan sequence (forty seconds per scan) instead of the more typical two hundred and forty seconds used in the dark-time observations with NIRSPEC. De-focusing can often occur rapidly during daytime observations due to sunlight striking and heating the telescope structure, so the focus was regularly checked and updated using a bright star close to the comet. The combination of seeing, drift, and de-focusing affect the measured point spread function (PSF); for our observations, the full width at half maximum (FWHM) of a stellar PSF was typically about four to eight pixels $\left(0^{\prime \prime} .8-1^{\prime \prime} .6\right)$ within the observing period (although there were likely brief periods of $2^{\prime \prime}$ or more). Additional observational details are given in 
Table 1

Observing log for observations of C/2012 S1 (ISON).

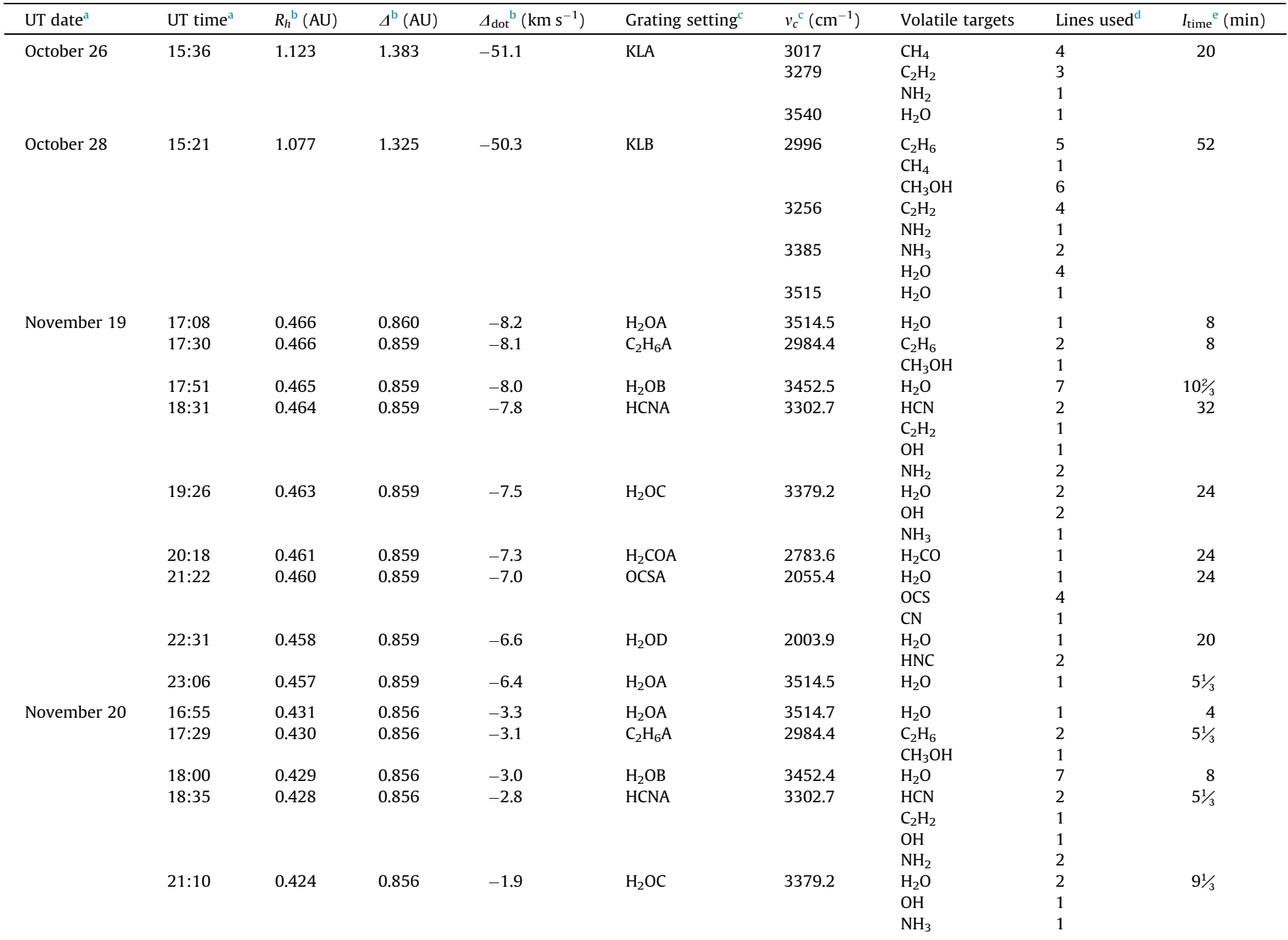

a Dates are UT 2013 and times are the midpoint within a range for the given grating setting. Observations on October 26 and 28 were obtained with NIRSPEC at the Keck Observatory. Observations on November 19 and 20 were obtained with CSHELL at the NASA IRTF.

b The heliocentric distance $\left(R_{h}\right)$, geocentric distance $(\Delta)$ and geocentric velocity $\left(\Delta_{\text {dot }}\right)$ of C/2012 S1 (ISON).

c NIRSPEC settings (KLA, KLB) contain multiple orders, so $v_{c}$ is the central wavenumber of a particular order within a setting. Each setting encompasses six orders with $\sim 40 \mathrm{~cm}^{-1}$ grasp per order. CSHELL settings have a spectral grasp of $\sim 5-9 \mathrm{~cm}^{-1}$, so $v_{c}$ is the central wavenumber of each CSHELL setting.

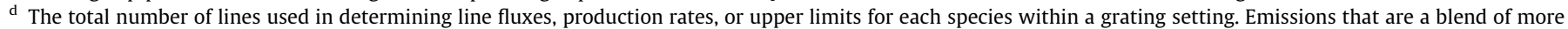
than one line are counted only once.

e On-source integration time.

Table 1. For each comet grating setting, spectra of infrared standard stars were obtained using the $4^{\prime \prime}$ wide slit.

The data were processed using algorithms specifically tailored to our comet observations, and application of these to both NIRSPEC and CSHELL have been described elsewhere (e.g., Dello Russo et al., 1998, 2006, 2008; DiSanti et al., 2001; Bonev, 2005). Initial processing included flat-fielding and removal of high-darkcurrent pixels and cosmic ray hits. Spectral frames were registered such that the spectral dimension fell along rows and the spatial dimension along columns. To account for drift between individual frames, each frame was registered to the same spectral row by using signal from the continuum before frames from the same grating setting were coadded.

Fully resolved atmospheric models covering the wavelength ranges of each grating setting were generated. These atmospheric models were binned to the instrumental sampling interval, convolved to the resolution of the comet spectrum, and normalized to the comet continuum and used to assign wavelength scales to the extracted spectra and to establish absolute column burdens for each significant absorbing species in the terrestrial atmosphere (Fig. 1). The column burdens for $\mathrm{H}_{2} \mathrm{O}$ within our best-fit atmospheric models were checked for reliability with contemporaneous atmospheric water opacity measurements recorded in the daily archive of the Caltech Sub-millimeter Observatory to assure consistency between grating settings. Volatile emission features were separated from the continuum by subtracting the normalized atmospheric model from the comet spectrum row by row, yielding the spectral residuals (residuals for each grating setting are shown at the bottom of each panel in Fig. 1 and also in Fig. 2). The atmospheric transmittance function is still convolved with the residuals so the true line flux $\left(F_{\text {line }}\right)$ incident at the top of the terrestrial atmosphere was determined by dividing the observed line flux by the monochromatic transmittance at the Doppler-shifted line position using the fully resolved, best-fit atmospheric models. Fluxes for individual lines sampled with NIRSPEC in October determined within 3-pixel (spectral) $\times 9$-pixel (spatial) extracts $\left(0^{\prime \prime} .43 \times 1^{\prime \prime} .72\right)$ centered on the peak gas intensity are listed in Table 2. Fluxes for individual lines sampled in November with 

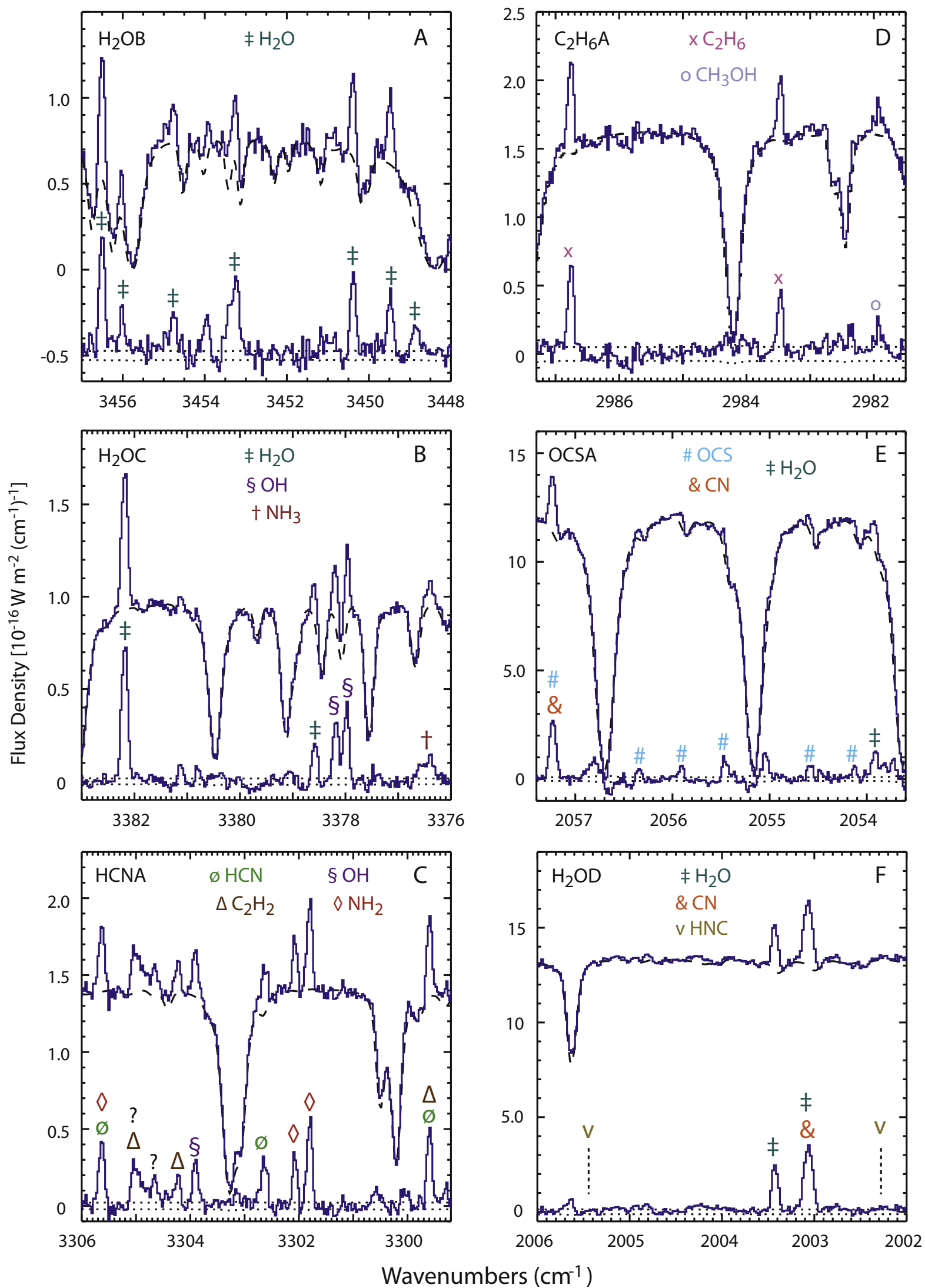

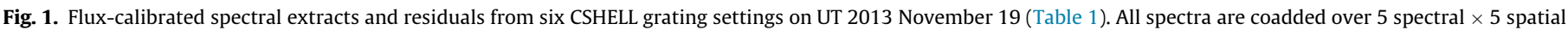

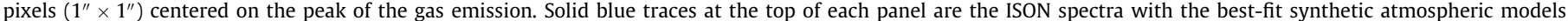

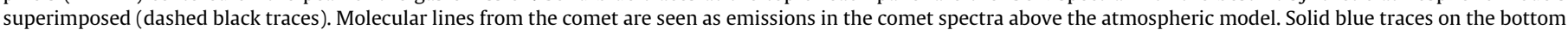

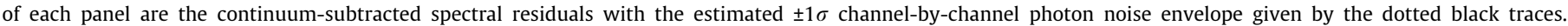

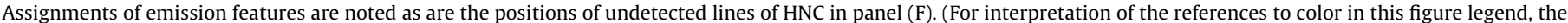
reader is referred to the web version of this article.) 

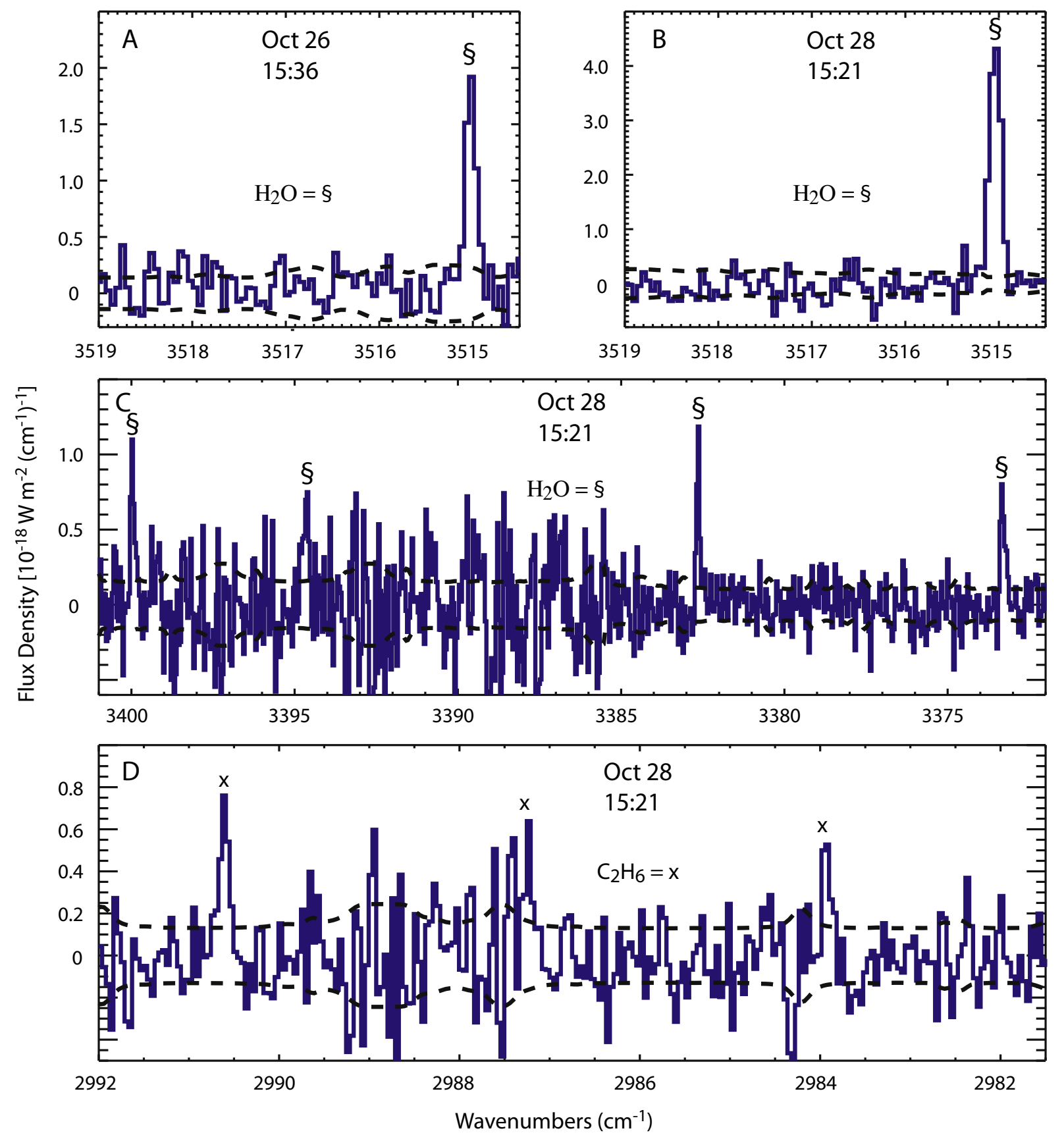

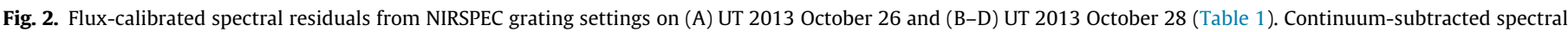

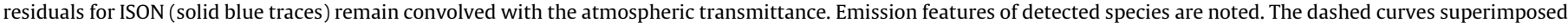

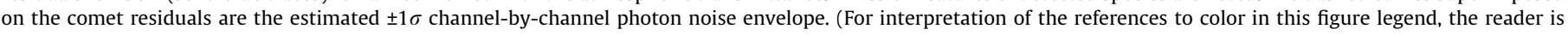
referred to the web version of this article.)

CSHELL and determined within 5-pixel (spectral) $\times 5$-pixel (spatial) extracts $\left(1^{\prime \prime} \times 1^{\prime \prime}\right)$ centered on the peak gas intensity are listed in Table 3.

Production rates and rotational temperatures were derived from the column densities within nucleus-centered extracts ( $3 \times 9$-pixels for NIRSPEC and $5 \times 5$-pixels for CSHELL) by applying a coma model that assumes spherically symmetric outflow and uniform velocity. Any outflow asymmetries will affect our derived global production rates, but this method has been shown to be a valid approach to first order and is unlikely to be a major source of uncertainty (Xie and Mumma, 1996). For ISON, we assume a gas outflow velocity of $v=0.8 \times R_{h}{ }^{-0.5} \mathrm{~km} \mathrm{~s}^{-1}$. This relationship is consistent with measured line widths seen at radio wavelengths at $R_{h}=0.67-0.58$ AU suggesting $v \sim 1.1 \mathrm{~km} \mathrm{~s}^{-1}$ (Agúndez et al., 2014), and from ALMA spectroscopy suggesting $v \sim 1.0 \mathrm{~km} \mathrm{~s}^{-1}$ at $R_{h}=0.54 \mathrm{AU}$ (Cordiner et al., 2014). For high-resolution infrared observations, the transit time for molecules from the nucleus to the edge of the field of view (in the plane of the sky) is generally short compared to the lifetime of measured species; in such cases the derived production rates are proportional to the assumed outflow velocity. However, for the CSHELL observations in November, as a result of the relatively small heliocentric distances, the transit time for molecules from the nucleus to the edge of the slit may have rivaled or exceeded the lifetimes of some species (e.g. $\mathrm{H}_{2} \mathrm{CO}$ and $\mathrm{NH}_{3}$ ), so in these cases uncertainties in the lifetime add to uncertainties in derived production rates. 
Table 2

Line fluxes in C/2012 S1 (ISON) from Keck/NIRSPEC observations.

\begin{tabular}{|c|c|c|c|c|c|c|c|}
\hline Molecule & Band ID & Line ID & Line pos. $\left(\mathrm{cm}^{-1}\right)^{\mathrm{a}}$ & $g$-factor $\left(10^{-7} \mathrm{~s}^{-1}\right)^{\mathrm{b}}$ & Line flux $\left(10^{-20} \mathrm{~W} \mathrm{~m}^{-2}\right)^{\mathrm{c}}$ & Growth factor $^{\mathrm{d}}$ & $Q\left(10^{25} \mathrm{~s}^{-1}\right)^{\mathrm{e}}$ \\
\hline \multicolumn{8}{|c|}{ UT 2013 October 26} \\
\hline $\mathrm{H}_{2} \mathrm{O}$ & $v_{1}+v_{3}-v_{1}$ & $2_{11}-3_{12}$ & 3514.41 & 13.6 & $72.9 \pm 7.5$ & $1.50 \pm 0.15$ & $870 \pm 120$ \\
\hline \multirow[t]{4}{*}{$\mathrm{CH}_{4}$} & $v_{3}$ & P2 & 2999.02 & 164 & $4.4 \pm 2.8$ & & $5.0 \pm 3.3$ \\
\hline & & Q1 & 3018.82 & 185 & $0.4 \pm 6.0$ & & $0.4 \pm 6.2$ \\
\hline & & RO & 3028.75 & 422 & $0.2 \pm 3.8$ & & $0.08 \pm 1.8$ \\
\hline & & $\mathrm{R} 1$ & 3038.50 & 316 & $1.9 \pm 2.5$ & & $1.1 \pm 1.5$ \\
\hline \multirow[t]{3}{*}{$\mathrm{C}_{2} \mathrm{H}_{2}$} & $v_{2}+v_{4}+v_{5}$ & P7 & 3265.26 & 127 & $1.6 \pm 2.8$ & & $2.2 \pm 3.9$ \\
\hline & & $\mathrm{R} 1$ & 3286.58 & 112 & $-2.2 \pm 1.8$ & & $-3.4 \pm 2.7$ \\
\hline & & R5 & 3295.88 & 108 & $3.1 \pm 2.9$ & & $5.0 \pm 4.6$ \\
\hline $\mathrm{NH}_{2}$ & $v_{1}$ & $2_{20}-1_{11}$ & 3301.71 & 200 & $5.7 \pm 1.6$ & & $5.7 \pm 3.1$ \\
\hline \multicolumn{8}{|c|}{ UT 2013 October 28} \\
\hline \multirow[t]{5}{*}{$\mathrm{H}_{2} \mathrm{O}$} & $v_{1}+v_{3}-v_{1}$ & $2_{11}-3_{12}$ & 3514.41 & 13.6 & $103 \pm 5$ & $1.36 \pm 0.14$ & $1010 \pm 114$ \\
\hline & $2 v_{1}-v_{3}$ & $1_{01}-2_{02}$ & 3399.37 & 2.10 & $19.2 \pm 2.5$ & & $1250 \pm 205$ \\
\hline & & $212-3_{13}$ & 3382.10 & 2.11 & $16.1 \pm 1.6$ & & $1050 \pm 149$ \\
\hline & & $22_{21}-3_{22}$ & 3372.75 & 1.07 & $12.9 \pm 1.3$ & & $1650 \pm 232$ \\
\hline & $2 v_{1}-v_{1}$ & $22_{21}-3_{30}$ & 3394.08 & 1.65 & $14.4 \pm 2.0$ & & $1200 \pm 205$ \\
\hline \multirow[t]{4}{*}{$\mathrm{NH}_{3}$} & $v_{1}$ & $\operatorname{sqR}(1,0)$ & 3376.29 & 28.6 & $-1.4 \pm 1.4$ & & $-6.7 \pm 6.6$ \\
\hline & & $\operatorname{sqR}(1,1)$ & & & & & \\
\hline & & $\operatorname{aqR}(2,0)$ & 3393.82 & 16.5 & $1.0 \pm 2.0$ & & $8.7 \pm 16.8$ \\
\hline & & $\operatorname{aqR}(2,1)$ & & & & & \\
\hline \multirow[t]{5}{*}{$\mathrm{C}_{2} \mathrm{H}_{6}$} & $v_{7}$ & ${ }^{\mathrm{R}} Q_{1}$ & 2990.09 & 352 & $9.7 \pm 1.4$ & & $4.27 \pm 0.76$ \\
\hline & & ${ }^{\mathrm{R}} Q_{0}$ & 2986.73 & 452 & $9.5 \pm 2.3$ & & $3.27 \pm 0.85$ \\
\hline & & ${ }^{\mathrm{P}} \mathrm{Q}_{1}$ & 2983.38 & 392 & $6.9 \pm 1.5$ & & $2.72 \pm 0.66$ \\
\hline & & ${ }^{\mathrm{P}} \mathrm{Q}_{2}$ & 2980.07 & 338 & $5.1 \pm 2.0$ & & $2.34 \pm 0.94$ \\
\hline & & ${ }^{\mathrm{P}} \mathrm{Q}_{3}$ & 2976.77 & 241 & $8.6 \pm 1.5$ & & $5.53 \pm 1.13$ \\
\hline $\mathrm{CH}_{4}$ & $v_{3}$ & P2 & 2999.02 & 164 & $3.4 \pm 1.7$ & & $3.26 \pm 1.68$ \\
\hline \multirow[t]{6}{*}{$\mathrm{CH}_{3} \mathrm{OH}$} & $v_{2}$ & $Q \mathrm{E}(-1 \rightarrow 0)$ & 3001.1 & 33 & $1.0 \pm 1.2$ & & $4.8 \pm 5.8$ \\
\hline & & $Q \mathrm{E}(0 \rightarrow-1)$ & 2999.8 & 23 & $0.4 \pm 1.4$ & & $2.6 \pm 9.6$ \\
\hline & & $Q \mathrm{E}(1 \rightarrow 0)$ & 2997.1 & 37 & $3.4 \pm 1.3$ & & $14 \pm 6$ \\
\hline & & $Q \mathrm{~A}(1 \rightarrow 0)$ & 2996.7 & 86 & $9.2 \pm 2.2$ & & $17 \pm 4$ \\
\hline & & $Q \mathrm{E}(1 \rightarrow 2)$ & 2990.8 & 37 & $1.1 \pm 1.4$ & & $4.6 \pm 5.8$ \\
\hline & & $Q \mathrm{~A}(2 \rightarrow 3)$ & 2981.8 & 29 & $2.2 \pm 1.7$ & & $12 \pm 9$ \\
\hline \multirow[t]{4}{*}{$\mathrm{C}_{2} \mathrm{H}_{2}$} & $v_{2}+v_{4}+v_{5}$ & P3 & 3274.82 & 169 & $1.7 \pm 1.9$ & & $1.5 \pm 1.6$ \\
\hline & & P5 & 3270.60 & 181 & $-1.6 \pm 2.1$ & & $-1.3 \pm 1.6$ \\
\hline & & P7 & 3265.26 & 127 & $1.1 \pm 1.3$ & & $1.2 \pm 1.4$ \\
\hline & & P9 & 3260.43 & 63 & $0.6 \pm 1.5$ & & $1.3 \pm 3.4$ \\
\hline $\mathrm{NH}_{2}$ & $v_{1}$ & 5 lines & 3249.9-3250.7 & 584 & $14.6 \pm 2.3$ & & $4.0 \pm 1.7$ \\
\hline
\end{tabular}

a These values are rest positions for single lines, average rest positions for two lines, or a range of rest positions for coadded lines.

b The $g$-factors at $R_{h}=1 \mathrm{AU}$ derived for an assumed rotational temperature of $50 \mathrm{~K}$.

c These values are line fluxes within a 3 spectral $\times 9$ spatial pixel extract $\left(0^{\prime \prime} .43 \times 1^{\prime \prime} .73\right)$ centered on the peak gas productivity. Uncertainties in fluxes include photon noise (reflected in the $\mathrm{S} / \mathrm{N}$ of individual lines) and an estimated uncertainty in the assumed rotational temperature of $\pm 10 \mathrm{~K}$.

d The multiplicative growth factor is the ratio between production rates derived from off-nucleus and on-nucleus extracts. On both dates the multiplicative growth factors for all species are assumed to be the same as was derived for the $\mathrm{H}_{2} \mathrm{O}$ line at $3514.41 \mathrm{~cm}^{-1}$.

e Derived production rates for individual lines. Uncertainties include photon noise (reflected in the S/N of individual lines) and uncertainties in the multiplicative growth factor. Calibration errors are not included.

Production rates derived from nucleus-centered extracts are always underestimated owing to slit losses, so a production rate growth curve analysis is performed to determine production rates from regions offset from the nucleus where slit losses due to seeing, comet drift, and suboptimal focus are less important (Dello Russo et al., 1998). For this analysis, the multiplicative growth factor is the ratio of derived production rates determined from off-nucleus extracts to production rates determined from extracts centered on the peak gas intensity. In cases where strong lines of a molecule are present, reliable multiplicative growth factors are derived. Reliable multiplicative growth factors can also be obtained when multiple emission lines from a single species are present within a grating setting and the signal-to-noise ratio of the combined molecular emission in offset positions from the nucleus is sufficient. For species whose lines are few, weak or not apparent (in cases where only upper limits are derived) multiplicative growth factors are assumed to be the same as for other species detected within the same grating setting.

It is expected that species that are produced with a significant contribution from an extended source will have a larger multiplicative growth factor than species released directly as a parent from the nucleus. However, seeing, tracking and de-focusing will also affect multiplicative growth factors, so these effects need to be accounted for when interpreting any differences between species. In particular, seeing, tracking and de-focusing were certainly variable between different settings obtained during daytime CSHELL observations on November 19 and 20. Fortunately molecules obtained within the same grating setting can be robustly compared, and for most settings lines of $\mathrm{H}_{2} \mathrm{O}$ or $\mathrm{OH}$ prompt emission were detected providing a measure of the $\mathrm{H}_{2} \mathrm{O}$ multiplicative growth factor and production rate. Multiplicative growth factors derived or assumed for individual emissions are given in Tables 2 and 3.

\section{Results}

\subsection{Rotational temperatures, production rates, and relative abundances}

In order to obtain rotational temperatures and production rates from measured line fluxes, the fluorescence efficiencies ( $g$-factors) for individual ro-vibrational lines are needed as a function of temperature. A description of the fluorescence models used in this analysis for all parent molecules is given elsewhere (e.g. Dello Russo et al., 2009a, 2011, and references therein). $\mathrm{NH}_{2}$ g-factors 
Table 3

Line fluxes in C/2012 S1 (ISON) from IRTF/CSHELL observations.

\begin{tabular}{|c|c|c|c|c|c|c|c|}
\hline Molecule & Band ID & Line ID & Line pos. ${ }^{\mathrm{a}}\left(\mathrm{cm}^{-1}\right)$ & $g$-factor ${ }^{\mathrm{b}}\left(10^{-7} \mathrm{~s}^{-1}\right)$ & Line flux ${ }^{\mathrm{c}}\left(10^{-18} \mathrm{~W} \mathrm{~m}^{-2}\right)$ & Growth factor $^{d}$ & $Q\left(10^{27} \mathrm{~s}^{-1}\right)^{\mathrm{e}}$ \\
\hline \multicolumn{8}{|c|}{ UT 2013 November 19} \\
\hline $\mathrm{H}_{2} \mathrm{O}$ & $v_{1}+v_{3}-v_{1}$ & $2_{11}-3_{12}$ & 3514.41 & 10.4 & $75.3 \pm 3.9$ & $2.29 \pm 0.05$ & $240 \pm 13$ \\
\hline \multirow[t]{2}{*}{$\mathrm{C}_{2} \mathrm{H}_{6}$} & \multirow{2}{*}{$v_{7}$} & ${ }^{\mathrm{R}} Q_{0}$ & 2986.73 & 305 & $7.34 \pm 0.51$ & $1.95 \pm 0.11$ & $0.80 \pm 0.07$ \\
\hline & & ${ }^{\mathrm{P}} Q_{1}$ & 2983.38 & 266 & $4.31 \pm 0.38$ & $2.31 \pm 0.11$ & $0.64 \pm 0.06$ \\
\hline $\mathrm{CH}_{3} \mathrm{OH}$ & $v_{2}$ & $Q \mathrm{~A}(2 \rightarrow 3)$ & 2981.8 & 29 & $2.53 \pm 0.36$ & $2.15 \pm 0.41$ & $3.18 \pm 0.75$ \\
\hline \multirow[t]{7}{*}{$\mathrm{H}_{2} \mathrm{O}$} & \multirow[t]{2}{*}{$v_{1}+v_{3}-v_{1}$} & $4_{22}-5_{23}$ & 3456.44 & 1.79 & $16.4 \pm 0.9$ & $1.95 \pm 0.10$ & $262 \pm 20$ \\
\hline & & $5_{24}-6_{25}$ & 3449.38 & 0.77 & $7.19 \pm 0.47$ & $1.75 \pm 0.19$ & $242 \pm 31$ \\
\hline & $v_{1}+v_{3}-v_{3}$ & $2_{21}-2_{20}$ & 3454.69 & 0.45 & $4.33 \pm 0.33$ & $2.39 \pm 0.52$ & $336 \pm 77$ \\
\hline & $2 v_{1}-v_{3}$ & $1_{10}-1_{11}$ & 3450.29 & 1.04 & $8.63 \pm 0.52$ & $2.01 \pm 0.15$ & $245 \pm 23$ \\
\hline & Blend & 2 lines & 3455.97 & 0.63 & $8.44 \pm 0.66$ & $1.85 \pm 0.16$ & $362 \pm 42$ \\
\hline & Blend & 2 lines & 3453.22 & 1.40 & $16.5 \pm 0.9$ & $1.74 \pm 0.17$ & $300 \pm 35$ \\
\hline & Blend & 3 lines & 3448.76 & 1.19 & $12.8 \pm 1.2$ & $2.10 \pm 0.19$ & $333 \pm 43$ \\
\hline \multirow[t]{2}{*}{$\mathrm{HCN}$} & \multirow[t]{2}{*}{$v_{3}$} & P3 & 3302.55 & 166 & $4.09 \pm 0.26$ & $2.11 \pm 0.10$ & $0.80 \pm 0.06$ \\
\hline & & $\mathrm{P} 4^{*}$ & 3299.53 & 199 & $3.62 \pm 0.23$ & $2.21 \pm 0.09$ & $0.62 \pm 0.05$ \\
\hline $\mathrm{C}_{2} \mathrm{H}_{2}$ & $v_{3}$ & R3 & 3304.17 & 99 & $2.26 \pm 0.18$ & $2.04 \pm 0.25$ & $0.72 \pm 0.11$ \\
\hline $\mathrm{OH}$ & $2 \rightarrow 1$ & P2. $52^{+} / 2^{-}$ & 3303.81 & & $3.19 \pm 0.22$ & $2.42 \pm 0.21$ & \\
\hline \multirow[t]{2}{*}{$\mathrm{NH}_{2}$} & \multirow[t]{2}{*}{$v_{1}$} & $\begin{array}{l}2_{20}-1_{11} \\
2.5 \rightarrow 1.5\end{array}$ & 3301.71 & 98 & $5.64 \pm 0.32$ & $3.05 \pm 0.18$ & $2.62 \pm 0.27$ \\
\hline & & $\begin{array}{l}2_{20}-1_{11} \\
1.5 \rightarrow 0.5\end{array}$ & 3301.99 & 51 & $2.96 \pm 0.20$ & & $2.65 \pm 0.29$ \\
\hline \multirow[t]{2}{*}{$\mathrm{H}_{2} \mathrm{O}$} & \multirow[t]{2}{*}{$2 v_{1}-v_{3}$} & $2_{12}-3_{13}$ & 3382.10 & 1.34 & $9.77 \pm 0.51$ & $2.36 \pm 0.05$ & $256 \pm 14$ \\
\hline & & $2_{02}-3_{03}$ & 3378.48 & 0.46 & $2.56 \pm 0.18$ & $3.14 \pm 0.28$ & $259 \pm 30$ \\
\hline \multirow[t]{2}{*}{$\mathrm{OH}$} & \multirow[t]{2}{*}{$1 \rightarrow 0$} & P4.5 2- & 3378.07 & & $4.16 \pm 0.25$ & $2.20 \pm 0.12$ & \\
\hline & & P4.5 2+ & 3377.89 & & $4.73 \pm 0.27$ & $2.11 \pm 0.11$ & \\
\hline $\mathrm{NH}_{3}$ & $v_{1}$ & $\begin{array}{l}\operatorname{sqR}(1,0) \\
\operatorname{sqR}(1,1)\end{array}$ & 3376.29 & 14.7 & $1.75 \pm 0.15$ & $5.75 \pm 0.65$ & $13.0 \pm 1.8$ \\
\hline $\mathrm{H}_{2} \mathrm{CO}$ & $v_{1}$ & 12 lines & 2781.0 & 123 & $4.59 \pm 0.31$ & $3.03 \pm 0.28$ & $2.92 \pm 0.33$ \\
\hline $\mathrm{H}_{2} \mathrm{O}$ & $v_{3}-v_{2}$ & $4_{04}-5_{05}$ & 2053.86 & 1.73 & $8.19 \pm 0.59$ & $2.59 \pm 0.30$ & $298 \pm 41$ \\
\hline \multirow[t]{4}{*}{ OCS } & \multirow[t]{4}{*}{$v_{1}$} & P19 & 2054.08 & 505 & $1.87 \pm 0.34$ & $3.16 \pm 0.30$ & $0.33 \pm 0.07$ \\
\hline & & P18 & 2054.53 & 523 & $3.43 \pm 0.33$ & & $0.58 \pm 0.08$ \\
\hline & & P15 & 2055.86 & 554 & $3.22 \pm 0.30$ & & $0.52 \pm 0.07$ \\
\hline & & P14 & 2056.30 & 555 & $2.83 \pm 0.32$ & & $0.45 \pm 0.07$ \\
\hline $\mathrm{CN}$ & $1 \rightarrow 0$ & R3 & 2057.20 & & $18.5 \pm 1.0$ & & \\
\hline $\mathrm{H}_{2} \mathrm{O}$ & $v_{1}-v_{2}$ & $1_{01}-2_{12}$ & 2003.39 & 3.71 & $19.5 \pm 1.2$ & $2.64 \pm 0.11$ & $343 \pm 26$ \\
\hline HNC & $v_{1}$ & P7 & 2002.22 & 218 & $1.99 \pm 0.63$ & & $0.52 \pm 0.16$ \\
\hline & & P6 & 2005.38 & 232 & $0.36 \pm 0.64$ & & $0.09 \pm 0.15$ \\
\hline $\mathrm{H}_{2} \mathrm{O}$ & $v_{1}+v_{3}-v_{1}$ & $2_{11}-3_{12}$ & 3514.41 & 10.4 & $97.2 \pm 4.9$ & $3.35 \pm 0.15$ & $440 \pm 30$ \\
\hline UT $2013 N$ & aber 20 & & & & & & \\
\hline $\mathrm{H}_{2} \mathrm{O}$ & $v_{1}+v_{3}-v_{1}$ & $2_{11}-3_{12}$ & 3514.41 & 9.44 & $146 \pm 7$ & $2.06 \pm 0.03$ & $410 \pm 22$ \\
\hline $\mathrm{C}_{2} \mathrm{H}_{6}$ & $v_{7}$ & ${ }^{\mathrm{R}} Q_{0}$ & 2986.73 & 277 & $12.1 \pm 0.9$ & $2.08 \pm 0.15$ & $1.37 \pm 0.14$ \\
\hline & & ${ }^{\mathrm{P}} Q_{1}$ & 2983.38 & 242 & $7.24 \pm 0.75$ & $2.60 \pm 0.20$ & $1.17 \pm 0.15$ \\
\hline $\mathrm{CH}_{3} \mathrm{OH}$ & $v_{2}$ & $Q \mathrm{~A}(2 \rightarrow 3)$ & 2981.8 & 29 & $4.16 \pm 0.73$ & $2.08 \pm 0.66$ & $5.0 \pm 1.8$ \\
\hline $\mathrm{H}_{2} \mathrm{O}$ & $v_{1}+v_{3}-v_{1}$ & $4_{22}-5_{23}$ & 3456.44 & 2.18 & $24.3 \pm 1.7$ & $2.52 \pm 0.15$ & $365 \pm 33$ \\
\hline & & $5_{24}-6_{25}$ & 3449.38 & 1.09 & $10.3 \pm 1.3$ & & $312 \pm 43$ \\
\hline & $v_{1}+v_{3}-v_{3}$ & $2_{21}-2_{20}$ & 3454.69 & 0.41 & $4.07 \pm 0.91$ & & $326 \pm 75$ \\
\hline & $2 v_{1}-v_{3}$ & $1_{10}-1_{11}$ & 3450.29 & 0.92 & $9.16 \pm 1.19$ & & $329 \pm 47$ \\
\hline & Blend & 2 lines & 3455.97 & 0.62 & $10.9 \pm 2.1$ & & $579 \pm 118$ \\
\hline & Blend & 2 lines & 3453.22 & 1.24 & $11.5 \pm 1.5$ & & $304 \pm 42$ \\
\hline & Blend & 3 lines & 3448.76 & 1.74 & $15.3 \pm 3.5$ & & $288 \pm 68$ \\
\hline $\mathrm{HCN}$ & $v_{3}$ & P3 & 3302.55 & 145 & $6.52 \pm 1.34$ & $2.20 \pm 0.44$ & $1.35 \pm 0.38$ \\
\hline & & $\mathrm{P} 4^{*}$ & 3299.53 & 176 & $4.51 \pm 1.29$ & & $0.77 \pm 0.27$ \\
\hline $\mathrm{C}_{2} \mathrm{H}_{2}$ & $v_{3}$ & R3 & 3304.17 & 88 & $2.23 \pm 1.35$ & & $0.76 \pm 0.51$ \\
\hline $\mathrm{OH}$ & $2 \rightarrow 1$ & $\mathrm{P} 2.52^{+} / 2^{-}$ & 3303.81 & & $6.65 \pm 1.54$ & & \\
\hline $\mathrm{NH}_{2}$ & $v_{1}$ & $\begin{array}{l}2_{20}-1_{11} \\
2.5 \rightarrow 1.5\end{array}$ & 3301.71 & 98 & $6.28 \pm 1.50$ & $3.05 \pm 0.61$ & $2.7 \pm 0.8$ \\
\hline & & $\begin{array}{l}2_{20}-1_{11} \\
1.5 \rightarrow 0.5\end{array}$ & 3301.99 & 51 & $2.99 \pm 1.49$ & & $2.5 \pm 1.3$ \\
\hline $\mathrm{H}_{2} \mathrm{O}$ & $2 v_{1}-v_{3}$ & $2{ }_{12}-3_{13}$ & 3381.10 & 1.20 & $13.3 \pm 1.0$ & $2.88 \pm 0.24$ & $417 \pm 48$ \\
\hline & & $2_{02}-3_{03}$ & 3378.48 & 0.44 & $5.62 \pm 0.96$ & $2.25 \pm 0.36$ & $379 \pm 88$ \\
\hline $\mathrm{OH}$ & $1 \rightarrow 0$ & P4.5 $2^{+} / 2^{-}$ & 3378 & & $15.2 \pm 1.4$ & $2.79 \pm 0.33$ & \\
\hline $\mathrm{NH}_{3}$ & $v_{1}$ & $\begin{array}{l}\operatorname{sqR}(1,0) \\
\operatorname{sqR}(1,1)\end{array}$ & 3376.29 & 12.6 & $3.59 \pm 0.76$ & $2.88 \pm 0.79$ & $14 \pm 5$ \\
\hline
\end{tabular}

\footnotetext{
a These values are rest positions for single lines, average rest positions for two lines, or a range of rest positions for coadded lines.
}

b The $g$-factors at $R_{h}=1 \mathrm{AU}$ are derived from a rotational temperature of $116 \mathrm{~K}$ on November 19 and $136 \mathrm{~K}$ on November 20 for all species (except $\mathrm{NH}_{2}$ ) based on derived rotational temperatures for $\mathrm{H}_{2} \mathrm{O}$ on these dates. Assumed $\mathrm{NH}_{2}$ rotational temperature is $80 \mathrm{~K}$ on both dates.

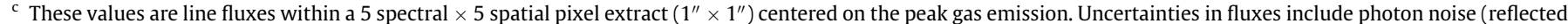
in the $\mathrm{S} / \mathrm{N}$ of individual lines).

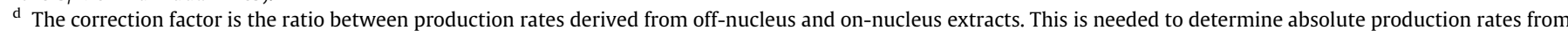

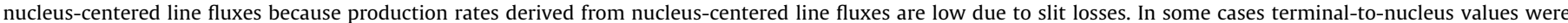

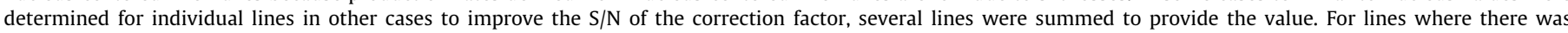
insufficient $\mathrm{S} / \mathrm{N}$ to determine a correction factor, one was assumed based on correction factors determined for lines within the same setting.

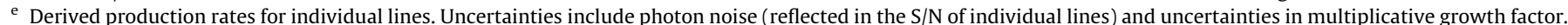
Calibration errors are not included. 
were obtained from a recently developed fluorescence model used to determine $\mathrm{NH}_{2}$ production rates in comets (Kawakita and Mumma, 2011).

Because the predicted strengths or $g$-factors for individual lines are temperature-dependent, the rotational temperature is needed for the most accurate determination of production rates. In general, the rotational temperature of a species in the coma can be determined if multiple strong lines with a range of ground-state rotational energies are measured (e.g. Dello Russo et al., 2004). In the case of ISON on October 26 and 28, few molecular lines were detected owing to the low productivity of the comet at that time (Fig. 1, Table 2), and a rotational temperature could not be reliably determined for any species. Therefore, $g$-factors for all sampled emissions are based on a common adopted rotational temperature of $50 \mathrm{~K}$ on both dates (Table 2). An assumed gas rotational temperature of $50 \mathrm{~K}$ on October 26 and 28 is reasonable based on comet productivity and heliocentric distance and is consistent with a measured rotational temperature of $\mathrm{H}_{2} \mathrm{O}$ in ISON of $(60 \pm 10) \mathrm{K}$ on November 7 when the comet was closer to the Sun and slightly more productive (Paganini et al., 2013).

The comet production rate increased significantly by midNovember, so a measure of the $\mathrm{H}_{2} \mathrm{O}$ rotational temperature was feasible with CSHELL observations. A grating setting that contains multiple $\mathrm{H}_{2} \mathrm{O}$ lines with a range of lower-state energies and which has been used in the past to provide sensitive $\mathrm{H}_{2} \mathrm{O}$ rotational temperature measurements (Dello Russo et al., 2004) was thus targeted on both IRTF dates. $\mathrm{H}_{2} \mathrm{O}$ rotational temperatures of $116 \pm 4$ and $136 \pm 9 \mathrm{~K}$ were derived on November 19 and 20 respectively, based on line fluxes within $5 \times 5$-pixel $\left(1^{\prime \prime} \times 1^{\prime \prime}\right)$ extracts centered on the peak gas intensity.

The $\mathrm{H}_{2} \mathrm{O}$ rotational temperature and column density were also determined spatially on November 19 by extracting emission spectra at successive positions along the slit where there was sufficient flux from measured $\mathrm{H}_{2} \mathrm{O}$ lines (Fig. 3). Spatially resolved rotational temperature determinations in C/2004 Q2 (Machholz) and 73P/Sc hwassmann-Wachmann 3 showed a steady decrease in the $\mathrm{H}_{2} \mathrm{O}$ rotational temperature as distance from the nucleus increased, consistent with sublimation from the nucleus followed by nearly adiabatic expansion of the outflowing $\mathrm{H}_{2} \mathrm{O}$ molecules (Bonev et al., 2007, 2008a). However, modeling of the coma of 73P/Schw assmann-Wachmann 3B predicted a steeper drop in both $\mathrm{H}_{2} \mathrm{O}$ column densities and rotational temperatures with increasing distance from the nucleus than seen in the observational data, suggesting a dominant release of $\mathrm{H}_{2} \mathrm{O}$ from a distributed source of icy grains in the coma (Fougere et al., 2012). Asymmetries between the $\mathrm{H}_{2} \mathrm{O}$ rotational temperature and $\mathrm{H}_{2} \mathrm{O}$ column densities were seen in 103P/Hartley 2, which may also be the result of significant release of $\mathrm{H}_{2} \mathrm{O}$ from icy grain sublimation in the coma (Bonev et al., 2013).

Spatially resolved rotational temperatures in the coma of ISON were measured on dates bracketing our November 19 observations and show a rapidly evolving coma between $R_{h}=0.53$ and $0.35 \mathrm{AU}$ (Bonev et al., 2014). The $\mathrm{H}_{2} \mathrm{O}$ rotational temperature at $0.53 \mathrm{AU}$ showed a steady decrease from a peak near the nucleus, whereas the rotational temperature at $R_{h}=0.35 \mathrm{AU}$ seemed to initially increase on both sides of the nucleus before a final decrease, giving a double-peaked appearance that bore no similarity to the spatial distribution for the $\mathrm{H}_{2} \mathrm{O}$ column density (Bonev et al., 2014). Observations reported here on November 19 at $R_{h}=0.465 \mathrm{AU}$ show a snapshot within this time of rapid coma evolution.

Spatially resolved rotational temperature measurements on November 19, obtained using the same methodology as Bonev et al. (2014), show a secondary peak in the rotational temperature at a projected distance of about $600 \mathrm{~km}$ from the nucleus in the antisolar direction, and coupled with the results from Bonev et al. (2014) suggests a growing spatial asymmetry of gas rotational temperature in the coma with decreasing heliocentric distance (Fig. 3). Detailed modeling is needed to definitively investigate the heating sources in the coma of ISON. Rotationally-hot water molecules released from icy grains in the coma can efficiently transfer energy through collisions to the cooler ambient gas (Fougere et al., 2012). Since ISON was likely ejecting large amounts of icy material from its nucleus during this time (Combi et al., 2014), this is a plausible scenario. However, at the small

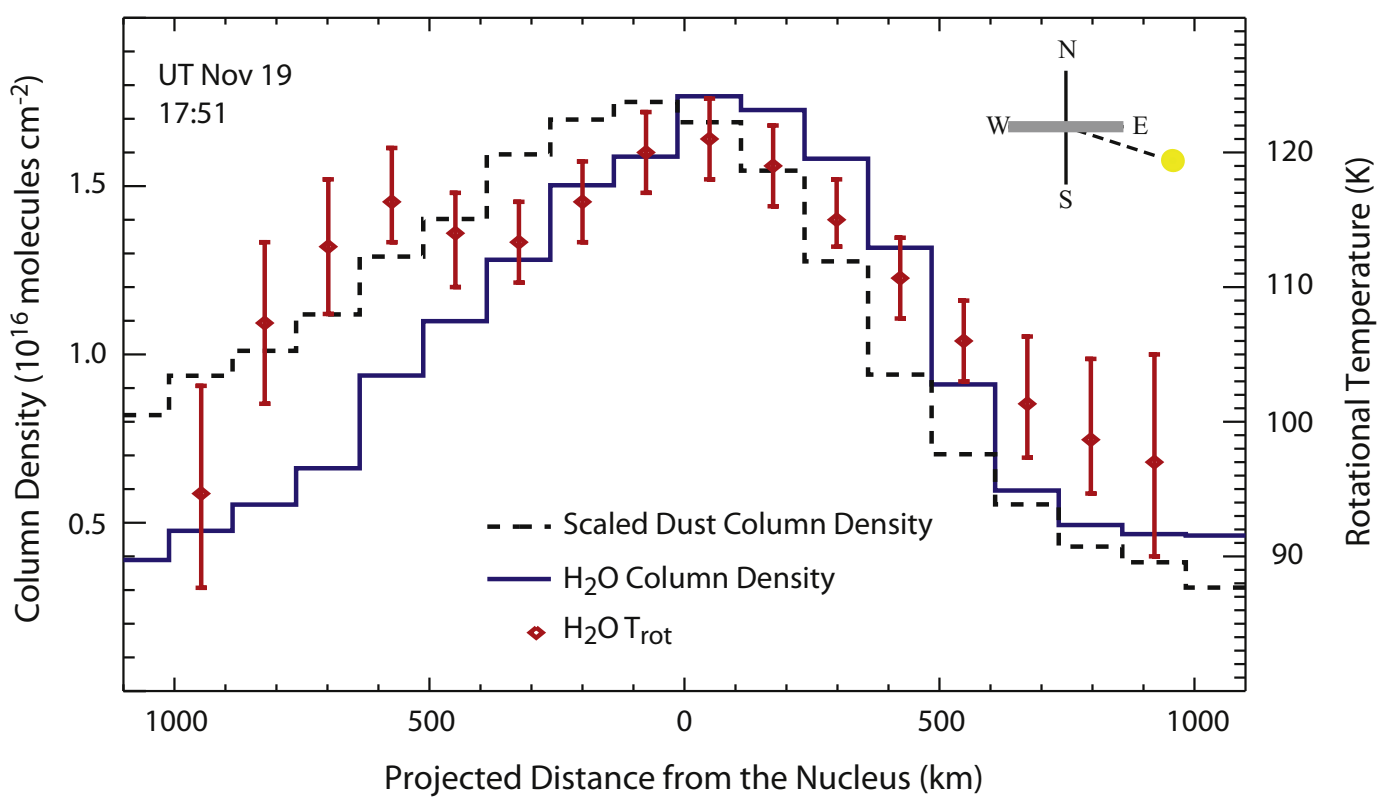

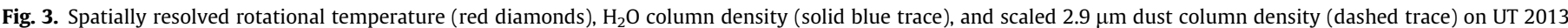

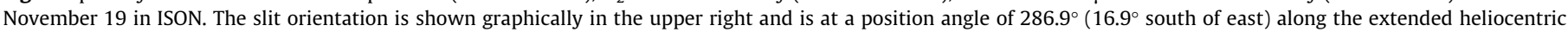

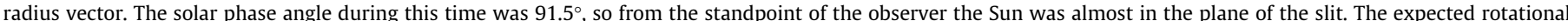

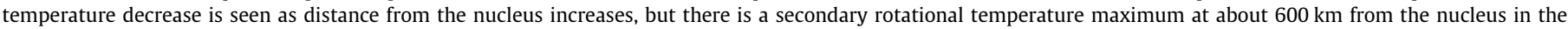
antisolar direction. (For interpretation of the references to color in this figure legend, the reader is referred to the web version of this article.) 
heliocentric distances of these observations, photochemical heating is also expected to have a significant effect on the temperature distribution in the coma (Combi et al., 1999). Thus the comparison of spatially resolved $\mathrm{H}_{2} \mathrm{O}$ column densities and rotational temperatures in ISON presented in this work and in Bonev et al. (2014) provide the most stringent observational constraint to date of how photochemical heating and icy grain release at small heliocentric distances work against cooling in the coma from adiabatic expansion.

Within the HCNA grating setting on November 19 (Table 1) there are potentially three strong $\mathrm{NH}_{2}$ lines, although one is blended with the P2 line of HCN (Fig. 4). Because the intensity of the blended $\mathrm{NH}_{2}$ line relative to the other two $\mathrm{NH}_{2}$ lines is extremely sensitive to rotational temperature, it is possible to estimate the rotational temperature of $\mathrm{NH}_{2}$ (Fig. 4A), assuming $\mathrm{NH}_{2}$ is populated by fluorescence in a Boltzmann distribution (see
Section 3.3.10). In the case of $\mathrm{C} / 2004 \mathrm{Q} 2$ (Machholz), the rotational temperature of $\mathrm{NH}_{2}$ was consistent with that of $\mathrm{H}_{2} \mathrm{O}$ suggesting that the $\mathrm{NH}_{2}$ population distribution was maintained by collisions with $\mathrm{H}_{2} \mathrm{O}$ (Kawakita and Mumma, 2011). A comparison of $\mathrm{NH}_{2}$ fluorescence models as a function of temperature with spectral residuals from the HCNA grating setting on November 19 suggests that the rotational temperature of $\mathrm{NH}_{2}$ is $\sim 80 \mathrm{~K}$ on November 19 and 20 (Fig. 4), which is significantly lower than the derived $\mathrm{H}_{2} \mathrm{O}$ rotational temperatures of $116 \pm 4$ and $136 \pm 9 \mathrm{~K}$ on these dates. The reason for this difference between $\mathrm{H}_{2} \mathrm{O}$ and $\mathrm{NH}_{2}$ rotational temperatures is unclear, but it suggests that the population distribution for $\mathrm{NH}_{2}$ in the coma of ISON is an intermediate case between a Boltzmann distribution maintained by collisions with $\mathrm{H}_{2} \mathrm{O}$ and a more rotationally relaxed distribution.

Because the $\mathrm{NH}_{2}$ rotational temperature is estimated by the relative intensities of only three emissions, and one is blended with

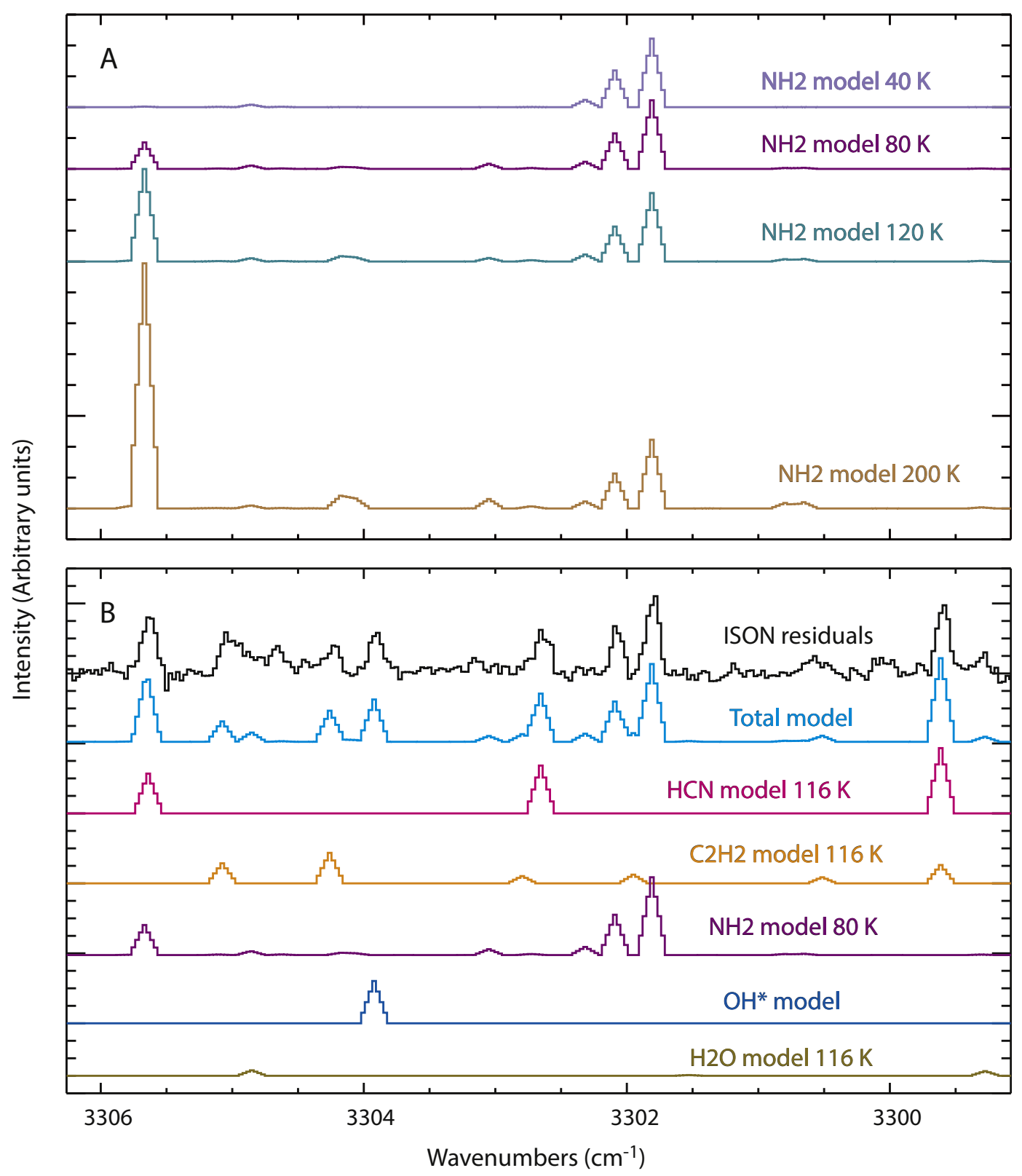

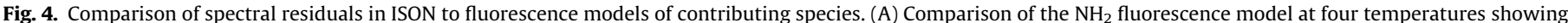

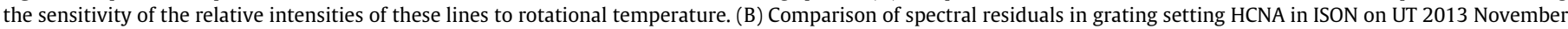
19 to individual fluorescence models for $\mathrm{HCN}, \mathrm{C}_{2} \mathrm{H}_{2}, \mathrm{NH}_{2}, \mathrm{OH}$, and $\mathrm{H}_{2} \mathrm{O}$, and to the combined model accounting for all species. 

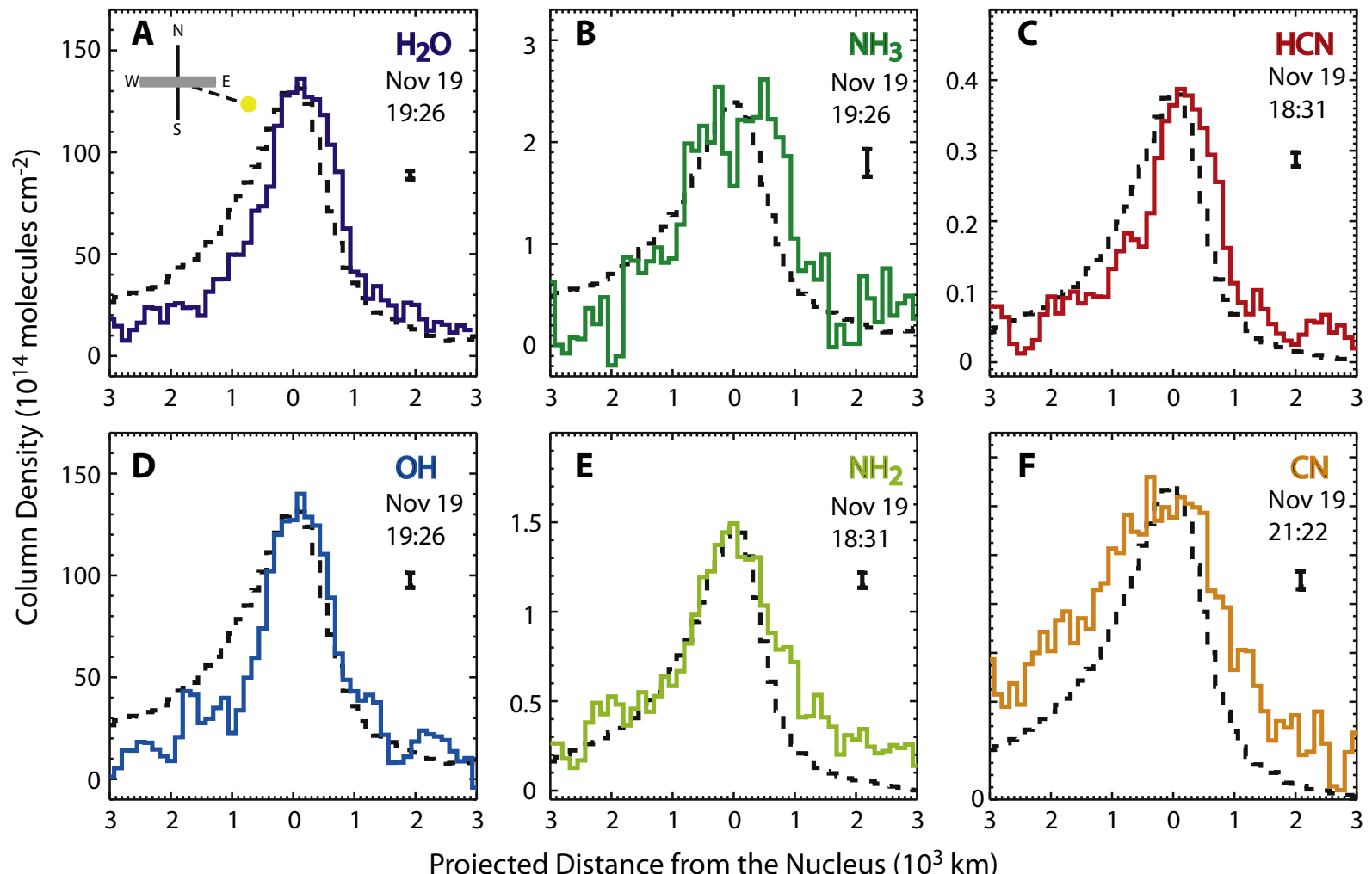

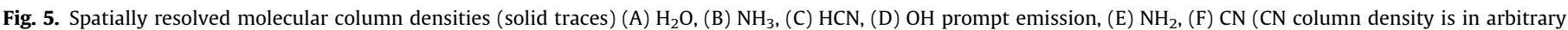

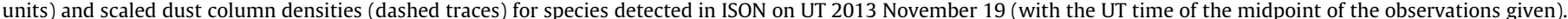

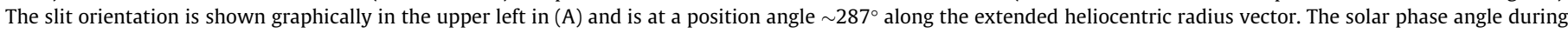

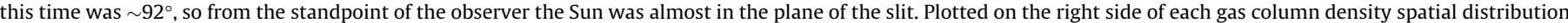

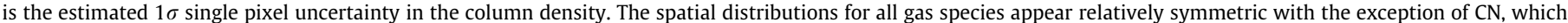
shows antisolar enhancement similar to dust.

the P2 line of $\mathrm{HCN}$, only coarse trends in the $\mathrm{NH}_{2}$ rotational temperature as a function of distance from the nucleus could be determined. $\mathrm{NH}_{2}$ rotational temperature appears to decrease by at least $20 \mathrm{~K}$ from the nucleus-centered position out to about $1000 \mathrm{~km}$ projected distance in the sunward direction, while it remains relatively constant from the nucleus-centered position out to about $1000 \mathrm{~km}$ projected distance in the antisolar direction. Although this is roughly similar to the spatially-resolved behavior of the $\mathrm{H}_{2} \mathrm{O}$ rotational temperature, we do not have the sensitivity to discern more subtle asymmetries or possible double-peaked structures for $\mathrm{NH}_{2}$ as were seen for $\mathrm{H}_{2} \mathrm{O}$ (Fig. 3).

Rotational temperatures for other species were not determined because either an insufficient number of spectral lines were detected or the relative intensities of the lines were not sufficiently sensitive to changes in the rotational temperature. Therefore, rotational temperatures of 116 and $136 \mathrm{~K}$ were adopted for all other species detected on November 19 and 20 respectively.

Line positions, $g$-factors, line fluxes, multiplicative growth factors, and production rates determined for individual lines are given in Tables 2 and 3. Production rates for each species are determined from a weighted average of production rates derived independently for all detected or expected lines. For species where upper limits are reported, a production rate was calculated independently over each line position for that species for which there are no known blends. Based on these line-by-line production rate determinations, the overall production rate or upper limit for each species on a particular date was determined (Table 4). Mixing ratios for each volatile species, expressed as a percentage with respect to $\mathrm{H}_{2} \mathrm{O}$, are determined on each date and also averaged over all dates (Table 4).

\subsection{Uncertainties in derived production rates and relative abundances}

Uncertainties in the derived rotational temperatures and absolute production rates are rarely dominated by the signal-to-noise ratios of individual spectral lines, as line-by-line deviations between the best-fit fluorescence model and the data are typically more important. Thus, reported uncertainties in line fluxes generally reflect the standard deviation from the mean of individual line measurements. Uncertainties for the multiplicative growth factors, flux calibration (estimated at 10\%), rotational temperature (estimated $\pm 10 \mathrm{~K}$ uncertainty for adopted values of $T_{\text {rot }}$ ), and photodissociation lifetimes for short-lived species were also included in absolute production rate uncertainties. Uncertainties in relative abundances for species that are sampled simultaneously with $\mathrm{H}_{2} \mathrm{O}$ (e.g. within the same grating setting) do not include flux calibration uncertainties.

In general, derived rotational temperatures for different species in comets are similar (e.g. $\mathrm{H}_{2} \mathrm{O}, \mathrm{C}_{2} \mathrm{H}_{6}, \mathrm{HCN}, \mathrm{CO}, \mathrm{H}_{2} \mathrm{CO}, \mathrm{CH}_{3} \mathrm{OH}, \mathrm{C}_{2} \mathrm{H}_{2}$ ) (e.g. Dello Russo et al., 2008, 2011; Mumma et al., 2011; DiSanti et al., 2014); however, for some species such as OCS and $\mathrm{NH}_{3}$, a reliable rotational temperature has not been measured at infrared wavelengths. We assume a rotational temperature of $116 \pm 10 \mathrm{~K}$ and $136 \pm 10 \mathrm{~K}$, with no variation as a function of distance from the nucleus, for all parent species on November 19 and 20 respectively (Table 4). It is possible that the rotational temperatures for other species are different from $\mathrm{H}_{2} \mathrm{O}$ (as seems to be the case for $\mathrm{NH}_{2}$ ), and this would affect the derived production rates (Table 5). Based on the particular lines sampled for each species on November 19 and 20, derived production rates for $\mathrm{HCN}, \mathrm{C}_{2} \mathrm{H}_{6}, \mathrm{C}_{2} \mathrm{H}_{2}$, and $\mathrm{NH}_{3}$ are more dependent on assumed rotational temperature than 

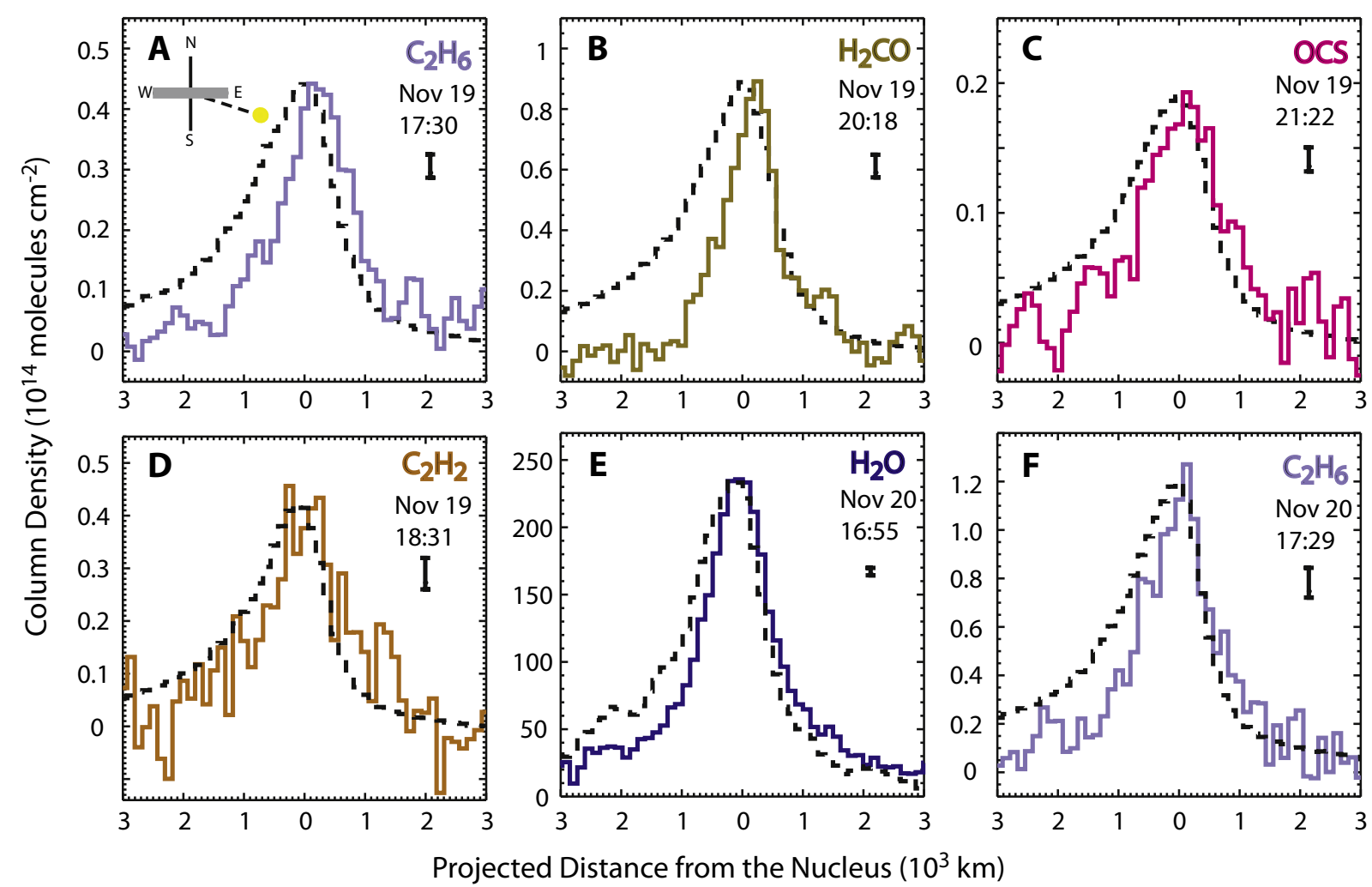

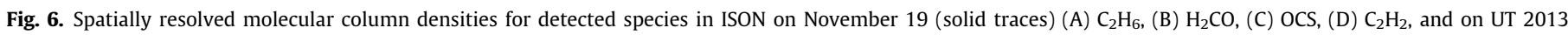

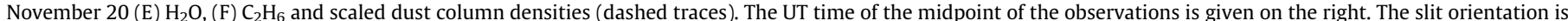

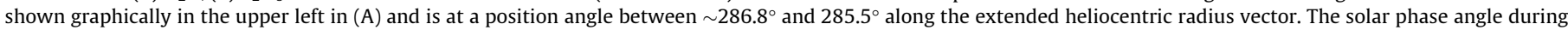

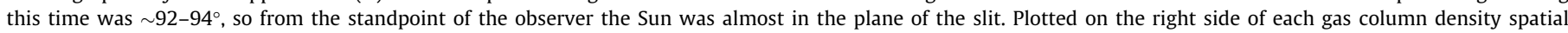

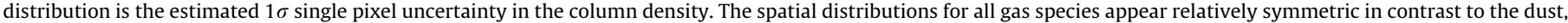
which shows an antisolar enhancement.

$\mathrm{H}_{2} \mathrm{O}, \mathrm{H}_{2} \mathrm{CO}$, OCS, and HNC (Table 5). Also, as discussed in Section 3.1, the derived $\mathrm{H}_{2} \mathrm{O}$ rotational temperature decreases with distance from the nucleus on November 19 (Fig. 3). If it is assumed that these species show the same rotational temperature trend with distance from the nucleus as seen for $\mathrm{H}_{2} \mathrm{O}$, we estimate that derived production rates and relative abundances in Table 4 would decrease by $\sim 4 \%$ for $\mathrm{C}_{2} \mathrm{H}_{6}, \mathrm{HCN}$ and $\mathrm{C}_{2} \mathrm{H}_{2}$ and $\sim 6 \%$ for $\mathrm{NH}_{3}$, and have no significant effect on derived production rates for $\mathrm{H}_{2} \mathrm{CO}$, OCS, and HNC.

The multiplicative growth factors for lines detected in October with NIRSPEC were determined from a strong $\mathrm{H}_{2} \mathrm{O}$ line with a rest position of $3514.41 \mathrm{~cm}^{-1}$ and assumed to be constant for all species with an uncertainty of $10 \%$ (Table 2). Growth factors were determined in the November CSHELL data from individual lines that were sufficiently strong or from a group of weaker lines within a single setting (Table 3). Uncertainties in multiplicative growth factors for lines detected with CSHELL range from $\sim 2 \%$ to $30 \%$ (Table 3 ) and can dominate the uncertainty in derived absolute production rates and mixing ratios for some species.

In most cases for observations of comets with CSHELL and NIRSPEC the photodissociation lifetime is long compared to the resident time of a molecule within the slit, allowing uncertainties in the photodissociation scale length to be ignored. However, in the case of ISON on November 19 and 20, lifetimes of some species are shorter or begin to rival the slit residence times, which can increase uncertainties in derived production rates if photodissociation rates are poorly constrained. For example, we assume a photodissociation lifetime for $\mathrm{H}_{2} \mathrm{CO}$ of $\sim 760 \mathrm{~s}$ on November 19 (Huebner et al., 1992); however, the derived $\mathrm{H}_{2} \mathrm{CO}$ production rate decreases by $\sim 20 \%$ if a photodissociation lifetime of $\sim 1060 \mathrm{~s}$ is used (Table 6; Bockelée-Morvan and Crovisier, 1992; Meier et al., 1993). Similarly, different lifetime estimates for $\mathrm{NH}_{3}$ and OCS affect derived production rates by $10 \%$ and $5 \%$ respectively on November 19 and 20 (Table 6). Different lifetime estimates for the other measured species with longer lifetimes do not significantly affect production rate determinations.

Finally, production rate calculations assume spherically symmetric uniform release directly from the nucleus, so contributions from extended sources can increase uncertainties. It is likely that in the case of species with short-lived progenitors that the effect on derived production rates are small. As mentioned in Section 2, the combination of seeing, drift, and de-focusing on the November dates result in a measured PSF of about four to eight pixels ( $\sim 500$ $1000 \mathrm{~km}$ in the spatial dimension), so the effects from short-lived progenitors (e.g. icy grains of $\mathrm{H}_{2} \mathrm{O}$, or $\mathrm{H}_{2} \mathrm{CO}$ parent) are already largely accounted for in the values and uncertainties of the multiplicative growth factors. The similar shape and the symmetry of the spatial distribution of species, with the exception of known daughter species $\mathrm{CN}$ support this (Figs. 5, 6).

\subsection{Temporal changes in activity and spatial distribution for volatiles in the coma}

Here we describe in detail the results for each individual molecule and, when appropriate, its relationship to other species. Although our data are limited to four dates, we also discuss the evolution of production rates and relative abundances with time and how relative abundances of species with respect to $\mathrm{H}_{2} \mathrm{O}$ compare to measurements in other comets. Finally, we discuss the nature of organic ices and dust in ISON as inferred from the spatial 
Table 4

Absolute and relative production rates and rotational temperatures in C/2012 S1 (ISON).

\begin{tabular}{|c|c|c|c|}
\hline Molecule & $T_{\text {rot }}(\mathrm{K})^{\mathrm{a}}$ & $Q\left(10^{26} \mathrm{~s}^{-1}\right)^{\mathrm{b}}$ & $\mathrm{Q} / \mathrm{Q}\left(\mathrm{H}_{2} \mathrm{O}\right) \%^{\mathrm{b}}$ \\
\hline \multicolumn{4}{|c|}{ UT 2013 October 26} \\
\hline $\mathrm{H}_{2} \mathrm{O}$ & 50 & $87 \pm 15$ & 100 \\
\hline $\mathrm{CH}_{4}$ & 50 & $<0.28$ & $<0.32$ \\
\hline $\mathrm{C}_{2} \mathrm{H}_{2}$ & 50 & $<0.40$ & $<0.46$ \\
\hline $\mathrm{NH}_{2}$ & 50 & $0.6 \pm 0.3$ & $0.7 \pm 0.4$ \\
\hline \multicolumn{4}{|c|}{ UT 2013 October 28} \\
\hline $\mathrm{H}_{2} \mathrm{O}$ & 50 & $110 \pm 20$ & 100 \\
\hline $\mathrm{C}_{2} \mathrm{H}_{6}$ & 50 & $0.35 \pm 0.07$ & $0.32 \pm 0.08$ \\
\hline $\mathrm{CH}_{4}$ & 50 & $<0.49$ & $<0.45$ \\
\hline $\mathrm{CH}_{3} \mathrm{OH}$ & 50 & $1.1 \pm 0.3$ & $1.0 \pm 0.3$ \\
\hline $\mathrm{C}_{2} \mathrm{H}_{2}$ & 50 & $<0.19$ & $<0.17$ \\
\hline $\mathrm{NH}_{3}$ & 50 & $<1.8$ & $<1.6$ \\
\hline $\mathrm{NH}_{2}$ & 50 & $0.40 \pm 0.18$ & $0.4 \pm 0.2$ \\
\hline \multicolumn{4}{|c|}{ UT 2013 November $19^{c}$} \\
\hline $\mathrm{H}_{2} \mathrm{O}$ & $116 \pm 4$ & $2700 \pm 300$ & 100 \\
\hline $\mathrm{C}_{2} \mathrm{H}_{6}$ & 116 & $7.1 \pm 1.0$ & $0.27 \pm 0.05$ \\
\hline $\mathrm{CH}_{3} \mathrm{OH}$ & 116 & $32 \pm 8$ & $1.2 \pm 0.3$ \\
\hline $\mathrm{H}_{2} \mathrm{CO}$ & 116 & $29 \pm 7$ & $1.1 \pm 0.3$ \\
\hline $\mathrm{HCN}$ & 116 & $6.8 \pm 1.0$ & $0.26 \pm 0.04$ \\
\hline $\mathrm{C}_{2} \mathrm{H}_{2}$ & 116 & $7.2 \pm 1.4$ & $0.27 \pm 0.05$ \\
\hline $\mathrm{NH}_{3}$ & 116 & $130 \pm 30$ & $5.0 \pm 1.2$ \\
\hline $\mathrm{NH}_{2}$ & 80 & $26 \pm 6$ & $1.0 \pm 0.2$ \\
\hline OCS & 116 & $4.6 \pm 0.7$ & $0.16 \pm 0.04$ \\
\hline HNC & 116 & $<4.4$ & $<0.13$ \\
\hline \multicolumn{4}{|c|}{ UT 2013 November $20^{c}$} \\
\hline $\mathrm{H}_{2} \mathrm{O}$ & $136 \pm 9$ & $3700 \pm 400$ & 100 \\
\hline $\mathrm{C}_{2} \mathrm{H}_{6}$ & 136 & $13 \pm 2$ & $0.35 \pm 0.06$ \\
\hline $\mathrm{CH}_{3} \mathrm{OH}$ & 136 & $50 \pm 19$ & $1.4 \pm 0.6$ \\
\hline $\mathrm{HCN}$ & 136 & $9.7 \pm 2.5$ & $0.26 \pm 0.07$ \\
\hline $\mathrm{C}_{2} \mathrm{H}_{2}$ & 136 & $7.6 \pm 5.1$ & $0.21 \pm 0.14$ \\
\hline $\mathrm{NH}_{3}$ & 136 & $140 \pm 60$ & $3.4 \pm 1.4$ \\
\hline $\mathrm{NH}_{2}$ & 80 & $27 \pm 9$ & $0.7 \pm 0.3$ \\
\hline
\end{tabular}

a The rotational temperature was assumed to be $50 \mathrm{~K}$ for all species on Octobe 26 and 28. The $\mathrm{H}_{2} \mathrm{O}$ rotational temperature was determined from the relative intensities of seven lines within setting $\mathrm{H}_{2} \mathrm{OB}$ on November 19 and 20 . The derived $\mathrm{H}_{2} \mathrm{O}$ rotational temperature was assumed for all other species on these dates except $\mathrm{NH}_{2}$, which was determined to be $\sim 80 \mathrm{~K}$.

$\mathrm{b}$ The uncertainties include standard error of the mean for individual line measurements, uncertainties in the multiplicative growth factor, an assumed $10 \%$ error in the flux calibration, and $\pm 10 \mathrm{~K}$ uncertainties in $T_{\text {rot }}$ for all assumed values.

c On November 19 and 20 listed $\mathrm{H}_{2} \mathrm{O}$ production rates are an average from all lines measured on that date. Relative abundances are compared to $\mathrm{H}_{2} \mathrm{O}$ values obtained from the same setting (or adjacent settings if no $\mathrm{H}_{2} \mathrm{O}$ lines are sampled).

distributions for species in the coma. We note that an independent infrared study of ISON using NIRSPEC and CSHELL was performed on several dates in October and November 2014 (DiSanti et al., in press), with derived production rates and mixing ratios in good agreement with results presented here, and providing more temporal coverage when results are combined.

\subsection{1. $\mathrm{H}_{2} \mathrm{O}$}

$\mathrm{H}_{2} \mathrm{O}$ lines are among the strongest seen in comets at infrared wavelengths and at least one line was detected on each of the four
Table 6

Photodissociation lifetimes for molecules in the coma of ISON (November 19).

\begin{tabular}{lc}
\hline Molecule & Photodissociation lifetime $(\mathrm{s})^{\mathrm{a}}$ \\
\hline $\mathrm{H}_{2} \mathrm{O}$ & $1.8 \times 10^{4}$ \\
$\mathrm{C}_{2} \mathrm{H}_{6}$ & $2.0 \times 10^{4}$ \\
$\mathrm{CH}_{3} \mathrm{OH}$ & $1.8 \times 10^{4}$ \\
$\mathrm{H}_{2} \mathrm{CO}$ & $7.6 \times 10^{2 \mathrm{~b}}$ \\
$\mathrm{HCN}$ & $1.6 \times 10^{4}$ \\
$\mathrm{C}_{2} \mathrm{H}_{2}$ & $1.5 \times 10^{4}$ \\
$\mathrm{NH}_{3}$ & $1.2 \times 10^{3 \mathrm{c}}$ \\
$\mathrm{NH}_{2}$ & $1.0 \times 10^{5}$ \\
$\mathrm{OCS}$ & $2.6 \times 10^{3}$ \\
$\mathrm{HNC}$ & $1.6 \times 10^{4 \mathrm{~d}}$ \\
$\mathrm{CN}$ & $6.6 \times 10^{4}$
\end{tabular}

a All photodissociation lifetimes from Huebner et al. (1992) with lifetimes at heliocentric distance $0.46 \mathrm{AU}$ determined by dividing lifetimes at $1 \mathrm{AU}$ by $R_{h}{ }^{-2}$.

b Bockelée-Morvan and Crovisier (1992) and Meier et al. (1993) give a longer lifetime of $\sim 1060 \mathrm{~s}$, which decreases the derived $\mathrm{H}_{2} \mathrm{CO}$ production rate on November 19 by $20 \%$.

c Allen et al. (1987) gives a longer lifetime of $\sim 1.4 \times 10^{3} \mathrm{~s}$, which decreases the derived $\mathrm{NH}_{3}$ production rates on November 19 and 20 by $\sim 10 \%$.

d The photodissociation rate of HNC is assumed to be the same as HCN.

dates allowing the determination of production rates. Individual line positions, $g$-factors, line fluxes, and derived production rates from each measured $\mathrm{H}_{2} \mathrm{O}$ line are given in Tables 2 and 3. The overall $\mathrm{H}_{2} \mathrm{O}$ production rates determined on each date are given in Table 4 . The combination of weather issues, lack of available time on source, and the relative low productivity of the comet during NIRSPEC observations on October 26 and 28 led to the detection of a limited number of $\mathrm{H}_{2} \mathrm{O}$ lines on these dates (Tables 1 and 2; Fig. 2A-C). Based on an assumed $\mathrm{H}_{2} \mathrm{O}$ rotational temperature of $50 \mathrm{~K}$, the derived $\mathrm{H}_{2} \mathrm{O}$ production rates on October 26.6 and 28.6 were $(8.7 \pm 1.5) \times 10^{27}$ and $(1.1 \pm 0.2) \times 10^{28}$ molecules $\mathrm{s}^{-1}$ respectively (Table 4). Derived water production rates from SOHO/SWAN were slightly higher during this time period $\left[Q_{\mathrm{H} 2 \mathrm{O}}=(2.79 \pm 0.20)\right.$ and $(1.93 \pm 0.40) \times 10^{28}$ molecules $\mathrm{s}^{-1}$ on October 25.9 and 28.9 respectively; Combi et al., 2014], whereas derived $\mathrm{H}_{2} \mathrm{O}$ production rates from $\mathrm{OH}$ detections at optical wavelengths with the TRAPPIST telescope (Jehin et al., 2011) are in good agreement $\left(Q_{\mathrm{H} 2 \mathrm{O}}=\right.$ $(6.8 \pm 3.1) \times 10^{27}$ molecules $\mathrm{s}^{-1}$ on October 26.4; Jehin and Opitom, personal communication] (Fig. 7A).

Our next observation of ISON was on November 19 and it was clear that the comet had brightened dramatically. We targeted multiple $\mathrm{H}_{2} \mathrm{O}$ lines near both 3 and $5 \mu \mathrm{m}$ (Tables 1 and 3, Fig. 1) and were able to detect lines of $\mathrm{H}_{2} \mathrm{O}$ or $\mathrm{OH}$ prompt emission in most CSHELL settings (Table 3; Fig. 1 ). The derived $\mathrm{H}_{2} \mathrm{O}$ production rates on November 19 and 20 were about 30-40 times higher than in late October (Table 4), and near contemporaneous SOHO/SWAN and TRAPPIST observations are in general agreement (Fig. 7A, Combi et al., 2014). However, the quickly evolving activity of ISON and the different techniques used to obtain $\mathrm{H}_{2} \mathrm{O}$ production rates make it difficult to compare measurements, even those obtained close in time. $\mathrm{H}_{2} \mathrm{O}$ production rate measurements from various techniques suggest that this $30-40$-fold increase between

Table 5

The dependence of derived production rates on rotational temperature (November 19).

\begin{tabular}{|c|c|c|c|c|c|}
\hline Molecule & $Q\left(10^{26} \mathrm{~s}^{-1}\right) T_{\text {rot }}=50 \mathrm{~K}$ & $Q\left(10^{26} \mathrm{~s}^{-1}\right) T_{\text {rot }}=70 \mathrm{~K}$ & $Q\left(10^{26} \mathrm{~s}^{-1}\right) T_{\text {rot }}=100 \mathrm{~K}$ & $Q\left(10^{26} \mathrm{~s}^{-1}\right) T_{\text {rot }}=116 \mathrm{~K}$ & $Q\left(10^{26} \mathrm{~s}^{-1}\right) T_{\text {rot }}=150 \mathrm{~K}$ \\
\hline $\mathrm{C}_{2} \mathrm{H}_{6}$ & $4.8 \pm 0.7$ & $5.5 \pm 0.8$ & $6.5 \pm 0.9$ & $7.1 \pm 1.0$ & $8.3 \pm 1.2$ \\
\hline $\mathrm{H}_{2} \mathrm{CO}$ & $45 \pm 8$ & $32 \pm 5$ & $29 \pm 5$ & $29 \pm 5$ & $31 \pm 5$ \\
\hline $\mathrm{HCN}$ & $3.8 \pm 0.6$ & $4.7 \pm 0.7$ & $6.1 \pm 0.9$ & $6.8 \pm 1.0$ & $8.4 \pm 1.2$ \\
\hline $\mathrm{C}_{2} \mathrm{H}_{2}$ & $4.6 \pm 0.9$ & $5.3 \pm 1.0$ & $6.5 \pm 1.3$ & $7.2 \pm 1.4$ & $8.7 \pm 1.7$ \\
\hline $\mathrm{NH}_{3}$ & $67 \pm 15$ & $85 \pm 20$ & $114 \pm 26$ & $130 \pm 30$ & $166 \pm 38$ \\
\hline $\mathrm{NH}_{2}$ & $13 \pm 3$ & $24 \pm 5$ & $32 \pm 7$ & $36 \pm 8$ & $45 \pm 10$ \\
\hline OCS & $4.5 \pm 0.7$ & $4.2 \pm 0.6$ & $4.4 \pm 0.7$ & $4.6 \pm 0.7$ & $5.1 \pm 0.8$ \\
\hline HNC & $<4.3$ & $<4.1$ & $<4.2$ & $<4.4$ & $<4.9$ \\
\hline
\end{tabular}



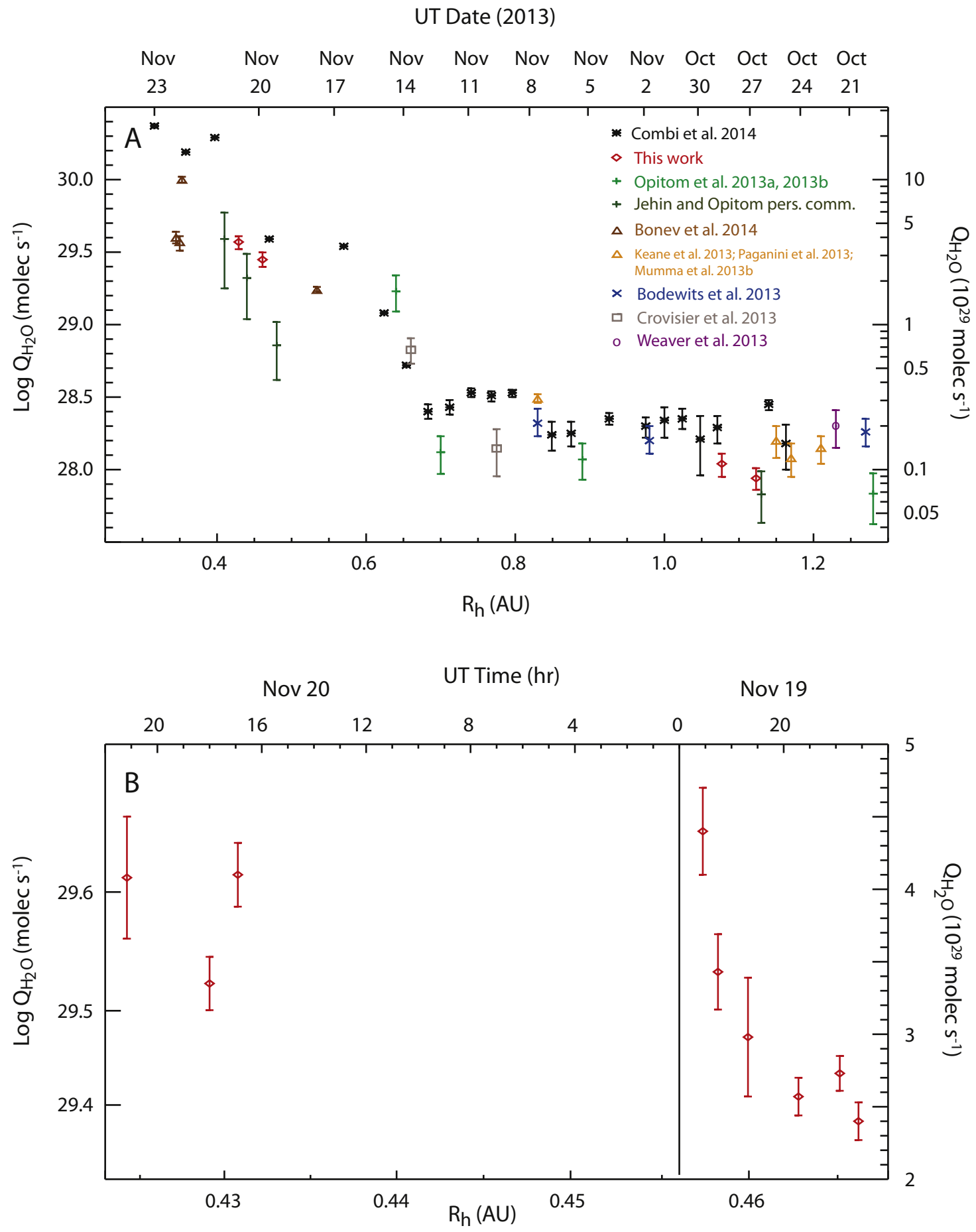

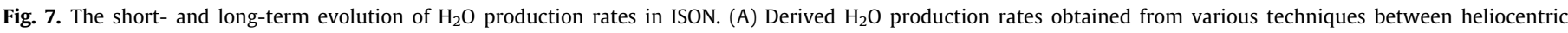

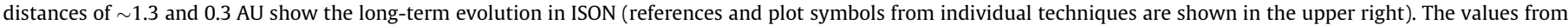

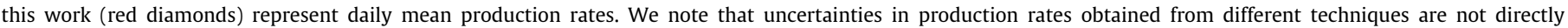

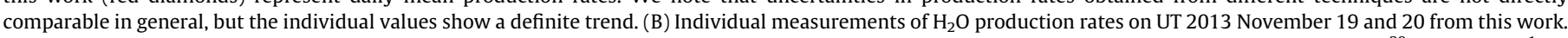

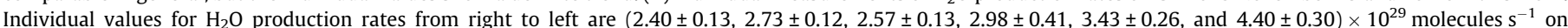

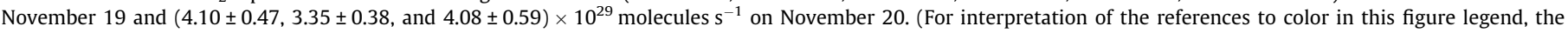
reader is referred to the web version of this article.) (See above-mentioned references for further information.)

$R_{h} \sim 1.1$ and 0.46 AU was not uniform but was characterized by little or no increase between $R_{h} \sim 1.3$ and $0.68 \mathrm{AU}$ (heliocentric dependence of $\sim R_{h}{ }^{-0.5}$ ) followed by a rapid increase of more than a factor of ten between about November 13 and November 19 with a continued steep increase between November 19 and November $23\left(R_{h}=0.32 \mathrm{AU}\right)$ (heliocentric dependence of $\sim R_{h}{ }^{-4}$ inside
$0.68 \mathrm{AU}$, Fig. 7A). Measurements obtained within $R_{h} \sim 0.68 \mathrm{AU}$ show that this increase was not steady but characterized by significant short-term variability (Fig. 7A). For example, $\mathrm{H}_{2} \mathrm{O}$ production rates measured with CSHELL decreased by almost a factor of three in less than $2 \mathrm{~h}$ on November 22 when ISON was at $R_{h}=0.35 \mathrm{AU}$ (Fig. 7A; Bonev et al., 2014). Measurements of $\mathrm{H}_{2} \mathrm{O}$ on November 
19 suggest that within uncertainties its production remained stable at $\sim 2.3-2.8 \times 10^{29}$ molecules s ${ }^{-1}$ between 17:00 and 19:45 with a steady increase beginning shortly thereafter until the final measurement of $(4.4 \pm 0.3) \times 10^{29}$ molecules s $^{-1}$ at 23:06 (Fig. 7B). Three measurements of the $\mathrm{H}_{2} \mathrm{O}$ production on November 20 between 16:55 and 21:10 show values that are consistent with those derived during the last two measurements on November 19 (Fig. 7B).

There was sufficient $\mathrm{S} / \mathrm{N}$ in detected $\mathrm{H}_{2} \mathrm{O}$ lines on November 19 and 20 to determine the spatial distribution for $\mathrm{H}_{2} \mathrm{O}$ in the coma. Compared to the spatial distribution for dust, which is asymmetric and shows an enhancement in the antisolar direction, the $\mathrm{H}_{2} \mathrm{O}$ spatial distribution appears symmetric with the peak column density slightly offset toward the sunward direction relative to the dust without notable spatial extension beyond that expected for a parent volatile released directly from the nucleus (Figs. 5A and 6E). Thus there is no evidence for significant release of $\mathrm{H}_{2} \mathrm{O}$ from icy grains outside a few hundred kilometers from the nucleus based on the shape of the $\mathrm{H}_{2} \mathrm{O}$ column density spatial distribution alone. However, the spatially resolved rotational temperature of $\mathrm{H}_{2} \mathrm{O}$ is asymmetric, showing a less rapid fall-off and a secondary peak $\sim 600 \mathrm{~km}$ projected distance from the nucleus in the antisolar direction (Fig. 3). This type of spatial asymmetry in the rotational temperature may be a signature of icy grain release on a scale that is not detectable in the column density spatial distribution for $\mathrm{H}_{2} \mathrm{O}$ (Bonev et al., 2013, 2014), so the possibility of an icy grain source for $\mathrm{H}_{2} \mathrm{O}$ is not ruled out.

3.3.2. $\mathrm{C}_{2} \mathrm{H}_{6}$

$\mathrm{C}_{2} \mathrm{H}_{6}$ is generally one of the easiest molecules to detect at infrared wavelengths owing to the strength of the $v_{7} Q$-branches and the moderately high abundances generally seen in comets (Dello Russo et al., 2001; Villanueva et al., 2011a). $\mathrm{C}_{2} \mathrm{H}_{6}$ was detected on three dates in ISON. Five $\mathrm{C}_{2} \mathrm{H}_{6} \mathrm{Q}$-branches were detected on October 28 (Table 2, Fig. 2D) with NIRSPEC, and individual positions, $g$-factors, fluxes, and derived production rates from each measured $Q$-branch are given in Table 2. The ${ }^{\mathrm{R}} Q_{0}$ and ${ }^{\mathrm{P}} \mathrm{Q}_{1}$ $Q$-branches of $\mathrm{C}_{2} \mathrm{H}_{6}$ were detected on November 19.6 and 20.6 with CSHELL (Fig. 1D), and individual positions, g-factors, fluxes, and derived production rates from each measured $Q$-branch are given in Table 3. The overall production rates for $\mathrm{C}_{2} \mathrm{H}_{6}$ determined on each date and mixing ratios with respect to $\mathrm{H}_{2} \mathrm{O}$ are given in Table 4. The mixing ratio of $\mathrm{C}_{2} \mathrm{H}_{6}$ with respect to $\mathrm{H}_{2} \mathrm{O}$ stayed constant on these three dates within measurement uncertainties, averaging $(0.31 \pm 0.04) \%$, which is depleted compared to the mean value of $\sim 0.6 \%$ measured in Oort cloud comets (Fig. 8; BockeléeMorvan et al., 2005; Mumma and Charnley, 2011).

The $Q$-branches were sufficiently strong to determine the spatial distribution of $\mathrm{C}_{2} \mathrm{H}_{6}$ along the slit on November 19 and 20. The $\mathrm{C}_{2} \mathrm{H}_{6}$ distribution appears similar to the spatial distribution for $\mathrm{H}_{2} \mathrm{O}$ - symmetric with the peak slightly offset in the sunward direction from the dust peak (Fig. 6A). There is no evidence for any significant contribution from an extended source of $\mathrm{C}_{2} \mathrm{H}_{6}$ in the coma of ISON.

\subsection{3. $\mathrm{CH}_{4}$}

$\mathrm{CH}_{4}$ is highly volatile, so its relative abundance in comets is likely influenced in some way by the formation temperature and processing history of cometary ices (Gibb et al., 2003; Kawakita et al., 2003, 2005). $\mathrm{CH}_{4}$ has a strong infrared fundamental band $\left(v_{3}\right)$ near $3.3 \mu \mathrm{m}$, which should make it one of the easier volatile species to detect in comets; however, $\mathrm{CH}_{4}$ has a strong terrestrial component that requires a sufficiently large geocentric Dopplershift to prevent atmospheric extinction of cometary lines. On October 26 and 28 the absolute value of the geocentric Doppler-shift of ISON ( $\Delta_{\text {dot }}$ in Table 1 ) was sufficiently large to target $\mathrm{CH}_{4} \cdot \mathrm{CH}_{4}$ was not detected in ISON on either date (Table 2) with $3 \sigma$ upper limits of $\mathrm{CH}_{4} / \mathrm{H}_{2} \mathrm{O}<0.32 \%$ and $0.45 \%$ on October 26 and 28 respectively (Table 4), which is in the depleted range compared to mean value of $\sim 0.8 \%$ measured in comets to date (Fig. 8; Gibb et al., 2003). The absolute geocentric Doppler-shift of ISON was not sufficiently large to enable a search for $\mathrm{CH}_{4}$ on November 19 or 20 .

\subsection{4. $\mathrm{CH}_{3} \mathrm{OH}$}

$\mathrm{CH}_{3} \mathrm{OH}$ lines are ubiquitous in cometary spectra in the wavelength range between about 3.30 and $3.56 \mu \mathrm{m}$ due to a mixture of weak to moderately strong $\mathrm{CH}_{3} \mathrm{OH}$ bands in this spectral region (e.g. Dello Russo et al., 2006; Villanueva et al., 2012; DiSanti et al., 2013). A tentative detection was obtained on October 28 based on flux from the positions of the six strongest $\mathrm{CH}_{3} \mathrm{OH}$ lines (Table 2). Relatively weak detections from a single line were used to determine production rates on November 19 and 20 (Table 3, Fig. 1D; $\mathrm{S} / \mathrm{N}$ of the $\mathrm{CH}_{3} \mathrm{OH}$ line was 7.0 and 5.7 on November 19 and 20 respectively). Because only a single line of $\mathrm{CH}_{3} \mathrm{OH}$ was targeted on November 19 and 20, the spatial distribution for $\mathrm{CH}_{3} \mathrm{OH}$ in the coma of ISON could not be reliably determined. The overall production rates for $\mathrm{CH}_{3} \mathrm{OH}$ determined on each date are given in Table 4. $\mathrm{A} \mathrm{CH}_{3} \mathrm{OH}$ production rate of $4.3 \times 10^{27}$ molecules s ${ }^{-1}$ was determined from IRAM observations of ISON on November 15.4 $\left(R_{h}=0.61 \mathrm{AU}\right.$; Agúndez et al., 2014), which is comparable to values obtained here $\left(3.2 \pm 0.8\right.$ and $5.0 \pm 1.9 \times 10^{27}$ molecules $^{-1}$ on November 19.7 and 20.7 respectively).

Mixing ratios for $\mathrm{CH}_{3} \mathrm{OH}$ with respect to $\mathrm{H}_{2} \mathrm{O}$ were measured on three dates remaining consistent within measurement uncertainties and averaging $(1.1 \pm 0.2) \%$ (Table 4$)$. Based on these measurements, $\mathrm{CH}_{3} \mathrm{OH}$ is depleted compared to the mean value of $\mathrm{CH}_{3} \mathrm{OH} / \mathrm{H}_{2} \mathrm{O} \sim 2 \%$ measured in other comets to date (Fig. 8; Mumma and Charnley, 2011). In contrast, the IRAM measurements on November 15.4 yield a mixing ratio $\mathrm{CH}_{3} \mathrm{OH} / \mathrm{HCN}=12$, which is in the normal range for comets and a factor of two to three higher than the mixing ratio derived from this work on November 19 and 20 of $\mathrm{CH}_{3} \mathrm{OH} / \mathrm{HCN}=4.8 \pm 1.2$. We note that other infrared spectral observations in late October and early November give much lower HCN production rates (Mumma et al., 2013a, 2013b; Paganini et al., 2013) and thus a $\mathrm{CH}_{3} \mathrm{OH} / \mathrm{HCN}$ ratio in agreement with the midNovember IRAM measurements. Because these observations occurred on different dates during a time when comet activity was changing rapidly, it is not clear if the differences in derived $\mathrm{CH}_{3} \mathrm{OH} / \mathrm{HCN}$ between IR and radio observations obtained several days apart in November reflect rapid changes in activity or composition in the coma of ISON, or some discrepancy in derived production rates between these methods.

\subsection{5. $\mathrm{H}_{2} \mathrm{CO}$}

Although $\mathrm{H}_{2} \mathrm{CO}$ has many observable lines between 3.42 and $3.64 \mu \mathrm{m}$ from the $v_{1}$ and $v_{5}$ fundamental bands, these bands are moderately weak and detections at infrared wavelengths are often difficult. Observations at radio wavelengths have identified comets with significant extended sources of $\mathrm{H}_{2} \mathrm{CO}$ (e.g. Biver et al., 1999); however, the weakness of the infrared lines of $\mathrm{H}_{2} \mathrm{CO}$ has generally limited IR detections to comets that exhibit a strong contribution from a native source (e.g. DiSanti et al., 2006). $\mathrm{H}_{2} \mathrm{CO}$ was detected in ISON on October $25\left(R_{h}=1.16 \mathrm{AU}\right)$ and November $7\left(R_{h}=0.83-\right.$ $A U)$ in another infrared study with NIRSPEC with derived abundances relative to $\mathrm{H}_{2} \mathrm{O}$ of $\sim 0.1-0.3 \%$ (DiSanti et al., in press). In this study, $\mathrm{H}_{2} \mathrm{CO}$ was detected on November 19.8 from emission near $2781 \mathrm{~cm}^{-1}$ due to a cluster of lines (Table 3 ), with a derived production rate of $(2.9 \pm 0.7) \times 10^{27}$ molecules $\mathrm{s}^{-1}$ and an abundance relative to $\mathrm{H}_{2} \mathrm{O}$ of $(1.1 \pm 0.3) \%$ (Table 4). This is in reasonable agreement with the derived production rate of $1.64 \times 10^{27}$ molecules $^{-1}$ from ALMA observations on November 17.5 considering the rapid increase and short-term variability in 


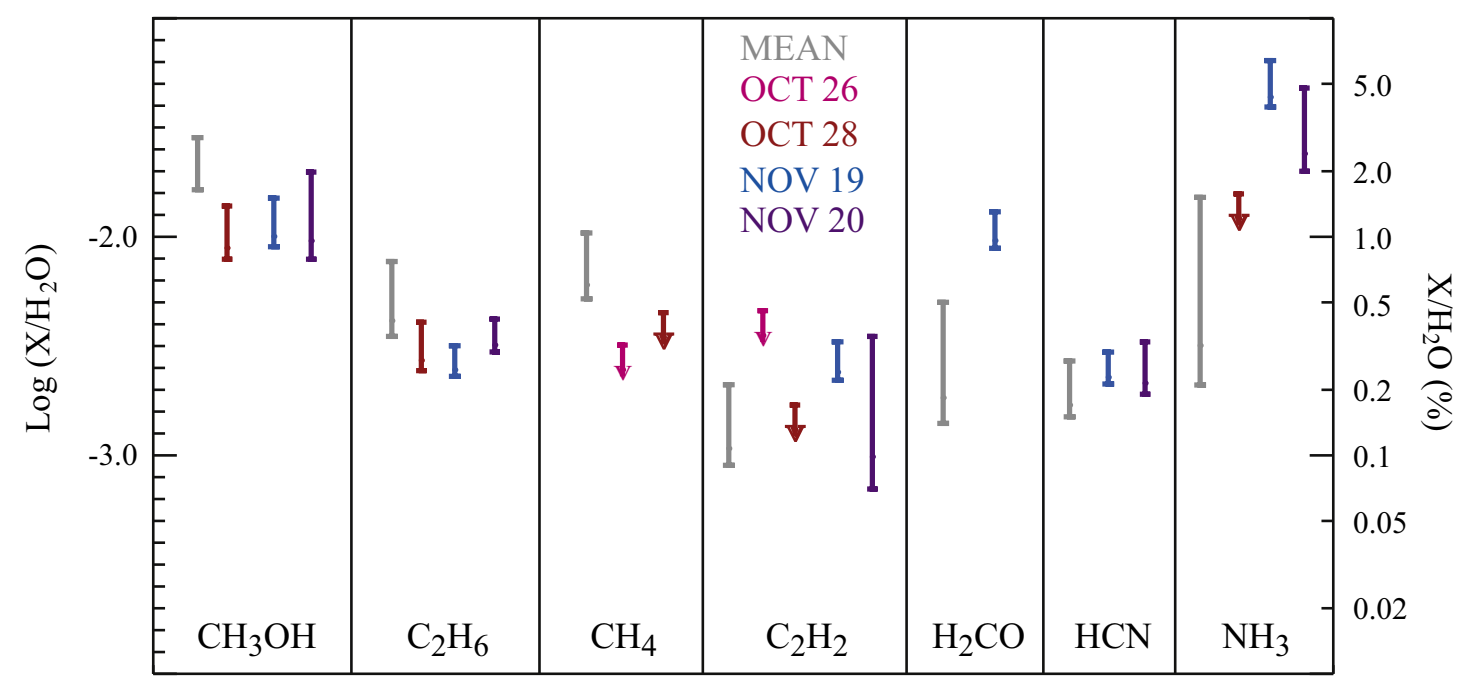

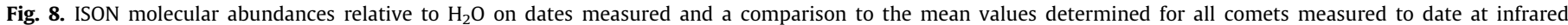
wavelengths (Bockelée-Morvan et al., 2005; Mumma and Charnley, 2011).

the productivity of ISON during this time (Cordiner et al., 2014). These infrared studies are suggestive of a significant increase in $\mathrm{H}_{2} \mathrm{CO} / \mathrm{H}_{2} \mathrm{O}$ between $R_{h}=0.83$ and $0.46 \mathrm{AU}$. The ratio of $\mathrm{H}_{2} \mathrm{CO} /$ $\mathrm{CH}_{3} \mathrm{OH}$ in ISON on November $19\left(\mathrm{H}_{2} \mathrm{CO} / \mathrm{CH}_{3} \mathrm{OH}=0.9 \pm 0.3\right)$ is one of the highest measured in a comet given that $\mathrm{H}_{2} \mathrm{CO} / \mathrm{CH}_{3} \mathrm{OH}$ ranges from $\sim 0.01$ to 1 over the population measured to date at infrared wavelengths (Bockelée-Morvan et al., 2005; Bonev et al., 2008b, 2009; Dello Russo et al., 2004, 2005, 2007, 2009a, 2011, 2014; DiSanti et al., 2002, 2006, 2014; Gibb et al., 2012; Mumma et al., 2001b, 2005; Mumma and Charnley, 2011; Paganini et al., 2012, 2014, 2015; Radeva et al., 2010, 2013; Villanueva et al., 2011b).

The strong $\mathrm{H}_{2} \mathrm{CO}$ emissions in ISON were sufficient to determine the spatial distribution along the slit on November 19 (Fig. 6B). The spatial distribution for $\mathrm{H}_{2} \mathrm{CO}$ appears relatively symmetric, with a shape that is narrower than spatial distributions for $\mathrm{H}_{2} \mathrm{O}, \mathrm{C}_{2} \mathrm{H}_{6}$ and dust and an offset from the dust spatial distribution consistent with $\mathrm{H}_{2} \mathrm{O}$ and $\mathrm{C}_{2} \mathrm{H}_{6}$ (Fig. $6 \mathrm{~B}$ ). The short photodissociation lifetime of $\mathrm{H}_{2} \mathrm{CO}$ likely contributes to its narrower spatial distribution. The lifetime of $\mathrm{H}_{2} \mathrm{CO}$ in the solar radiation field at $R_{h}=0.46 \mathrm{AU}$ is $\sim 760$ s (Huebner et al., 1992), which is about a factor of twenty less than that of $\mathrm{H}_{2} \mathrm{O}$ and $\mathrm{C}_{2} \mathrm{H}_{6}$ and shorter than the time it would take a molecule of $\mathrm{H}_{2} \mathrm{CO}$ to traverse the projected spatial field-ofview along the CSHELL slit. The symmetric and narrow spatial distribution of $\mathrm{H}_{2} \mathrm{CO}$ appears consistent with its primary release as a parent ice; however, observations with ALMA that also show a symmetric and narrow spatial distribution of $\mathrm{H}_{2} \mathrm{CO}$ similar to $\mathrm{HCN}$ additionally suggest a significant contribution from a distributed source of $\mathrm{H}_{2} \mathrm{CO}$ in ISON on November 17.5 with a parent scale length of 230-330 km (Cordiner et al., 2014). Our measurements cannot rule out a significant contribution from an extended source of $\mathrm{H}_{2} \mathrm{CO}$ as suggested by Cordiner et al. (2014) because the short parent scale length is within our seeing disk on November 19. The observed $\mathrm{H}_{2} \mathrm{CO}$ spatial distribution is symmetric and shows no extension in the dust-enhanced antisolar direction, therefore, any parent is likely short-lived (volatile or organic grain) and not associated with the micron-sized dust seen in the coma.

Cordiner et al. (2014) suggested that the high abundance of $\mathrm{H}_{2} \mathrm{CO}$ in ISON at small heliocentric distances could be the result of more rapid breakdown of an organic precursor material, which is consistent with an increase in $\mathrm{H}_{2} \mathrm{CO} / \mathrm{H}_{2} \mathrm{O}$ as ISON approached perihelion. Alternatively, the increasing $\mathrm{H}_{2} \mathrm{CO} / \mathrm{H}_{2} \mathrm{O}$ and $\mathrm{H}_{2} \mathrm{CO} /$ $\mathrm{CH}_{3} \mathrm{OH}$ with decreasing $R_{h}$ may reflect temporal variability in the chemistry of the sublimating ices. Relative abundances of $\mathrm{H}_{2} \mathrm{CO}$ and $\mathrm{CH}_{3} \mathrm{OH}$ ices may be controlled by the efficiency of hydrogen atom addition reactions (Fuchs et al., 2009). The possible increase of $\mathrm{H}_{2} \mathrm{CO} / \mathrm{CH}_{3} \mathrm{OH}$ in ISON as it approached perihelion may coincide with the rapidly accelerating removal of radiation-processed outer layers that are poor in $\mathrm{H}_{2} \mathrm{CO}$ and the sampling of a larger fraction of more pristine $\mathrm{H}_{2} \mathrm{CO}$-rich ices from this dynamically new comet.

\subsubsection{OCS}

Analysis of dust in Comet $1 \mathrm{P} /$ Halley suggested that most cometary sulfur is contained within grains (Jessberger et al., 1988). In general, $\mathrm{H}_{2} \mathrm{~S}$ is the dominant sulfur-bearing volatile in comets with typical derived abundances at radio wavelengths $\mathrm{H}_{2} \mathrm{~S} / \mathrm{H}_{2} \mathrm{O} \sim 1 \%$ (Bockelée-Morvan et al., 2005). OCS is also an important source of volatile sulfur and has been detected in comets at both infrared and radio wavelengths (Woodney et al., 1997; Dello Russo et al., 1998; Bockelée-Morvan et al., 2000). The spatial distribution of OCS in C/1995 01 (Hale-Bopp) suggested that a significant amount of OCS was produced from an extended source, likely from grains (Dello Russo et al., 1998).

We detected four lines of OCS in ISON on November 19.9 (Table 3) with a derived production rate of $(4.6 \pm 0.7) \times 10^{26}$ molecules $\mathrm{s}^{-1}$ and $\mathrm{OCS} / \mathrm{H}_{2} \mathrm{O}=(0.16 \pm 0.04) \%$ (Table 4$)$, which is a typical abundance compared to the few comets where OCS has been previously measured (Bockelée-Morvan et al., 2005). The OCS emissions were sufficiently strong when coadded to determine the spatial distribution along the slit on November 19, and the spatial distribution of OCS appears similar in shape and has a similar offset relative to the dust as $\mathrm{H}_{2} \mathrm{O}$ and $\mathrm{C}_{2} \mathrm{H}_{6}$ (Fig. 6C). Despite these similarities there is evidence that a significant amount of OCS may come from an extended source in the coma of ISON. The photodissociation lifetime of OCS in the solar radiation field at $R_{h}=0.46 \mathrm{AU}$ is $\sim 2600 \mathrm{~s}$ (Huebner et al., 1992), which is at least twice as long as $\mathrm{H}_{2} \mathrm{CO}$, but almost a factor of ten less than that of $\mathrm{H}_{2} \mathrm{O}$ and $\mathrm{C}_{2} \mathrm{H}_{6}$; therefore, the OCS spatial distribution appears broader than expected based on its short photodissociation lifetime. Any extended source of OCS would likely come from volatiles or grains not associated with the micron-sized dust seen in the coma., More rigorous analysis that is beyond the scope of the present work is needed to reveal the existence and properties of any extended source of OCS in ISON.

\subsection{7. $\mathrm{C}_{2} \mathrm{H}_{2}$}

The presence of $\mathrm{C}_{2} \mathrm{H}_{2}$ in comets is of potential astrobiological importance as reactions of $\mathrm{CN}$ and $\mathrm{C}_{2} \mathrm{H}$ radicals with unsaturated hydrocarbons such as $\mathrm{C}_{2} \mathrm{H}_{2}$ can form complex organics and amino 
acids (Kaiser and Balucani, 2002). $C_{2}$ has been measured for decades in many comets at optical wavelengths, and its relative abundance has served as a proxy for the carbon-chain chemistry in comets (e.g. A'Hearn et al., 1995; Fink, 2009; Cochran et al., 2012). The parent sources of $C_{2}$ in comets are not completely understood and likely vary in importance within the comet population. Molecules measured at infrared wavelengths such as $\mathrm{C}_{2} \mathrm{H}_{6}$ and $\mathrm{C}_{2} \mathrm{H}_{2}$ are potentially important sources of $\mathrm{C}_{2}$ in comets. Although $\mathrm{C}_{2} \mathrm{H}_{6}$ is generally more abundant in comets than $\mathrm{C}_{2} \mathrm{H}_{2}$, evidence suggests that $\mathrm{C}_{2} \mathrm{H}_{2}$ is likely the more important source of $\mathrm{C}_{2}$ in comets. Whereas the photodissociation efficiency of $\mathrm{C}_{2} \mathrm{H}_{2}$ to $\mathrm{C}_{2}$ is close to $100 \%$, only a small fraction of $\mathrm{C}_{2} \mathrm{H}_{6}$ photodissociates to $C_{2}$ (Helbert et al., 2005) with an efficiency perhaps close to $0 \%$ (Weiler, 2011). Comparison of production rates in comets also suggests an inefficient photodissociation efficiency of $\mathrm{C}_{2} \mathrm{H}_{6}$ to $\mathrm{C}_{2}$ of $<20 \%$ (Dello Russo et al., 2009a). Although $\mathrm{C}_{2} \mathrm{H}_{2}$ is likely the major volatile parent of $C_{2}$, in some comets the spatial distribution is flatter than expected from simple photodissociation of a parent molecule, so a contribution from organic grains may be significant in many comets (Combi and Delsemme, 1986; Combi and Fink, 1997).

$\mathrm{C}_{2} \mathrm{H}_{2}$ was targeted on all dates but only definitively detected on November 19.8. On October 28 the $\mathrm{C}_{2} \mathrm{H}_{2}$ production rate was $<1.9 \times 10^{25}$ molecules $\mathrm{s}^{-1}$ with $\mathrm{C}_{2} \mathrm{H}_{2} / \mathrm{H}_{2} \mathrm{O}<0.17 \%$. A single line of $\mathrm{C}_{2} \mathrm{H}_{2}$ was detected on November 19 with a derived production rate of $(7.2 \pm 1.4) \times 10^{26}$ molecules $\mathrm{s}^{-1}$, indicating that the $\mathrm{C}_{2} \mathrm{H}_{2}$ production rate had increased by more than a factor of thirty since October 28 (Table 4). The derived mixing ratio on November 19 was $\mathrm{C}_{2} \mathrm{H}_{2} / \mathrm{H}_{2} \mathrm{O}=(0.27 \pm 0.05) \%$, which is slightly enhanced compared to the mean values seen in comets (Fig. 8). It is also higher than the derived $3 \sigma$ upper limit on October 28, suggesting a possible increase in $\mathrm{C}_{2} \mathrm{H}_{2} / \mathrm{H}_{2} \mathrm{O}$ as ISON approached perihelion (Fig. 8); however, a lack of temporal coverage precludes any definite conclusion. A complete summary of production rates and mixing ratios for $\mathrm{C}_{2} \mathrm{H}_{2}$ is given in Table 4 .

It is interesting to compare $\mathrm{C}_{2} \mathrm{H}_{2}$ and $\mathrm{C}_{2} \mathrm{H}_{6}$ abundances, which may provide clues on the formation environment or chemical evolution of ISON. With almost all measured values to date between $\sim 0.1$ and $1, \mathrm{C}_{2} \mathrm{H}_{2} / \mathrm{C}_{2} \mathrm{H}_{6}$ abundances in comets show less variability than seen between most other cometary molecules (Bonev et al., 2008b, 2009; Dello Russo et al., 2002, 2004, 2005, 2007, 2008, 2009a, 2009b, 2011, 2014; DiSanti et al., 2014; Gibb et al., 2012; Kobayashi et al., 2010; Magee-Sauer et al., 2002; Mumma et al., 2001a, 2001b, 2005; Paganini et al., 2012, 2014, 2015; Radeva et al., 2010, 2013; Villanueva et al., 2011b). Although temporal coverage is sparse, relative abundances of $\mathrm{C}_{2} \mathrm{H}_{2}$ and $\mathrm{C}_{2} \mathrm{H}_{6}$ appear to increase from an upper-limit in the typical range of $\mathrm{C}_{2} \mathrm{H}_{2} / \mathrm{C}_{2} \mathrm{H}_{6}<0.53$ on October 28 to one of the highest values measured in a comet at $R_{h}=0.46 \mathrm{AU}\left(\mathrm{C}_{2} \mathrm{H}_{2} / \mathrm{C}_{2} \mathrm{H}_{6}=1.0 \pm 0.3\right)$ (Table 4). $\mathrm{C}_{2} \mathrm{H}_{6}$ can form from hydrogen atom addition reactions on $\mathrm{C}_{2} \mathrm{H}_{2}$ ice (Hiraoka et al., 2000); however, $\mathrm{C}_{2} \mathrm{H}_{2}$ is not efficiently formed from ion or ultraviolet processing of cometary ice analogs (Colangeli et al., 2005). The increasing $\mathrm{C}_{2} \mathrm{H}_{2} / \mathrm{C}_{2} \mathrm{H}_{6}$ and $\mathrm{H}_{2} \mathrm{CO} / \mathrm{CH}_{3} \mathrm{OH}$ (see Section 3.3.5) with decreasing $R_{h}$ may suggest that ISON contained more pristine natal cloud material that was subjected to less processing from hydrogen atom addition reactions in the nebula compared to other comets. The possible increase of $\mathrm{C}_{2} \mathrm{H}_{2} / \mathrm{C}_{2} \mathrm{H}_{6}$ in ISON as it approached perihelion may coincide with the increased sampling of more pristine layers deeper within the comet after the initial preferential removal of most of the radiation-processed outer layers. It is worth noting that the high $\mathrm{C}_{2} \mathrm{H}_{2} / \mathrm{C}_{2} \mathrm{H}_{6}$ measured in ISON on November 19 is similar to $\mathrm{C}_{2} \mathrm{H}_{2} / \mathrm{C}_{2} \mathrm{H}_{6}=(0.96 \pm 0.17)$ measured in the dynamically new Comet C/2006 P1 (McNaught) at $R_{h}=0.55 \mathrm{AU}$ (Dello Russo et al., 2009b).

Optical observations show about a factor of three increase in $\mathrm{C}_{2} / \mathrm{H}_{2} \mathrm{O}$ between $R_{h}=1.16 \mathrm{AU}$ (October 25 ) and $0.44 \mathrm{AU}$ (November 20) as ISON approached perihelion (McKay et al., 2014). Although some caution is warranted in comparing $\mathrm{C}_{2}$ and $\mathrm{C}_{2} \mathrm{H}_{2}$ observations obtained with different techniques and considering the short-term variability in ISON at this time, such comparisons can still be useful for showing large-scale differences. Optical observations provided measurements of $\mathrm{C}_{2} / \mathrm{H}_{2} \mathrm{O}$ near the time of our observations that are in agreement with each other $[\sim 0.7 \%$ on November 15 with ARCES at the Apache Point Observatory (McKay et al., 2014)], and $\sim 1 \%$ on November 19 and 20 with the TRAPPIST telescope, but are higher than the $\mathrm{C}_{2} \mathrm{H}_{2} / \mathrm{H}_{2} \mathrm{O}$ measured on November 19 by about a factor of three, suggesting that at this time $\mathrm{C}_{2} \mathrm{H}_{2}$ was not the dominant source of $\mathrm{C}_{2}$. Based on previous compositional measurements in comets it is unlikely that another volatile ice could provide a major source of $\mathrm{C}_{2}$; therefore, the majority of $\mathrm{C}_{2}$ seen in ISON is likely derived from the sublimation of organic grains. The spatial distribution of $\mathrm{C}_{2} \mathrm{H}_{2}$ in the coma of ISON was obtained on November 19, and it appears symmetric and offset from the dust in a manner similar to spatial distributions for $\mathrm{H}_{2} \mathrm{O}$ and $\mathrm{C}_{2} \mathrm{H}_{6}$, suggesting that most $\mathrm{C}_{2} \mathrm{H}_{2}$ seen in the coma is released directly from ices in the nucleus with no measurable contribution from an extended source (Fig. 6D).

\subsection{8. $\mathrm{HCN}$}

$\mathrm{HCN}$ is one of the most easily detected molecules in comets at both infrared and radio wavelengths, and its mixing ratio with respect to $\mathrm{H}_{2} \mathrm{O}$ within the comet population shows less variation compared to most other measured volatile species (BockeléeMorvan et al., 2005). HCN is routinely observed in the infrared through its strong $v_{3}$ band near $3.0 \mu \mathrm{m}$ (e.g. Magee-Sauer et al., 1999).

HCN was detected on November 19.8 and 20.8 by targeting three lines (P2, P3, and P4) within a single CSHELL grating setting. The $\mathrm{P} 4 \mathrm{HCN}$ line is blended with a line of $\mathrm{C}_{2} \mathrm{H}_{2}$ whose contribution is generally both easy to correct for and minor (Fig. 4B). The P2 and P3 lines are generally free from contamination; however, in comets with high abundances of $\mathrm{NH}_{2}$ such as ISON (see Section 3.3.9), there can be significant and difficult-to-quantify blending of $\mathrm{NH}_{2}$ within the P2 line (Fig. 4). Therefore, only the P3 and P4 lines were used to determine the $\mathrm{HCN}$ abundance (Table 3). The derived HCN production rates on these dates are given in Table 4 . HCN production rates determined from the IRAM telescope between November 13.4 and November 16.4 (Agúndez et al., 2014) and ALMA on November 17.5 (Cordiner et al., 2014) are generally lower than the values derived here on November 19.8 and 20.8. We note that direct comparisons are difficult because the productivity of ISON was highly variable during this time (Agúndez et al., 2014) and HCN production rates determined at radio wavelengths are often lower than those derived contemporaneously in the infrared (e.g. Magee-Sauer et al., 2008).

The mixing ratio of $\mathrm{HCN}$ with respect to $\mathrm{H}_{2} \mathrm{O}$ was consistent on November 19 and 20 within measurement uncertainties, averaging $(0.26 \pm 0.03) \%$, which is within the range of mean values measured in other comets (Table 4; Fig. 8). HCN was not targeted during our October time with NIRSPEC due to time and weather constraints; however, other NIRSPEC observations on October 25 and November 7 derived $\mathrm{HCN} / \mathrm{H}_{2} \mathrm{O}<0.1 \%$ and $(0.06 \pm 0.02) \%$ respectively (Mumma et al., 2013a; Paganini et al., 2013), which are consistent with updated derived values in DiSanti et al. (in press). These measurements suggest an increase in $\mathrm{HCN} / \mathrm{H}_{2} \mathrm{O}$ of about a factor of four between $R_{h}=0.83 \mathrm{AU}$ (November 7) and 0.46 AU (November 19). Hydrogen atom addition reactions can readily deplete $\mathrm{HCN}$ on grains (Theule et al., 2011; Bast et al., 2013), as can polymerization initiated by reactions with free radicals (e.g. Rettig et al., 1992). Therefore, the increasing $\mathrm{HCN} / \mathrm{H}_{2} \mathrm{O}$ in ISON as it approached perihelion may be further evidence for a transition from sampling processed outer layers to sampling increasingly more primitive natal 
material that was formerly protected from alteration deep within the nucleus.

Comparison of $\mathrm{HCN}$ to $\mathrm{CN}$ production rates should be done cautiously considering the different techniques used and he short-term variability in ISON as it approached perihelion, but such comparisons can reveal global trends. Optical observations also measured an increase of about a factor of five in $\mathrm{CN}$ production during this time (McKay et al., 2014), but the magnitude and perhaps also the evolution of $\mathrm{HCN}$ and $\mathrm{CN}$ production was different. The measured $\mathrm{CN} / \mathrm{H}_{2} \mathrm{O}$ increased rapidly from $\sim 0.15 \%$ on October $25\left(R_{h}=1.16 \mathrm{AU}\right)$ to $\sim 0.55 \%$ on November $6\left(R_{h}=0.87 \mathrm{AU}\right)$ and $\sim 0.75 \%$ on November 15 ( $R_{h}=0.62 \mathrm{AU}$ ) (McKay et al., 2014). A similar increase in $\mathrm{CN} / \mathrm{H}_{2} \mathrm{O}$ was also measured from TRAPPIST telescope observations between $R_{h}=1.13$ and $0.41 \mathrm{AU}$, with $\mathrm{CN} / \mathrm{H}_{2} \mathrm{O}$ increasing from $\sim 0.2 \%$ to $0.8 \%$ (Jehin and Opitom, personal communication). Thus, $\mathrm{CN}$ production appears to be about a factor of ten higher than HCN when the comet was at $R_{h} \sim 0.83-0.87 \mathrm{AU}$ (November 6-7), but only about a factor of three higher at $R_{h}=0.46 \mathrm{AU}$. We note that $\mathrm{H}_{2} \mathrm{O}$ production rates derived in this work are in good agreement with $\mathrm{H}_{2} \mathrm{O}$ production rates obtained at optical wavelengths close in time, so it suggests that these differences in $\mathrm{HCN}$ and $\mathrm{CN}$ evolution are real and not due to a disparity in $\mathrm{H}_{2} \mathrm{O}$ production rates determined from different techniques (Fig. 7). Whereas the increase of $\mathrm{CN}$ production appears steady between $R_{h}=1.16$ and $0.62 \mathrm{AU}$, the evolution of HCN suggests that most of its increase in productivity relative to $\mathrm{H}_{2} \mathrm{O}$ occurred inside $R_{h}=0.83 \mathrm{AU}$. Although these measurements suggest a different temporal evolution of $\mathrm{HCN}$ and $\mathrm{CN}$ release in ISON, there are important caveats. These conclusions are based on only a few measurements. In addition, because only an upper limit for $\mathrm{HCN} / \mathrm{H}_{2} \mathrm{O}$ was determined on October 25 (Mumma et al., 2013a; DiSanti et al., in press), we cannot rule out the possibility that $\mathrm{HCN} / \mathrm{H}_{2} \mathrm{O}$ was several times smaller than the value determined on November 7 (Paganini et al., 2013) and thus steadily increasing during this time similar to $\mathrm{CN}$.

Comparison of $\mathrm{HCN}$ and $\mathrm{CN}$ production rates alone indicate that $\mathrm{HCN}$ is only a minor contributor to the total CN in ISON, with most $\mathrm{CN}$ coming from another source. It is also possible that a significant amount of HCN may be produced from an extended source as ISON approached the Sun as suggested by the steep increase in $\mathrm{HCN} / \mathrm{H}_{2} \mathrm{O}$ between 0.83 and $0.46 \mathrm{AU}$. In addition to $\mathrm{HCN}$, emission lines of $\mathrm{CN}$ were also detected in our infrared spectra of ISON (Fig. 1E and F). To date models for $\mathrm{CN}$ emission at infrared wavelengths have not been developed to the point that reliable $\mathrm{CN}$ production rates can be determined; however, the spatial distribution of HCN and $\mathrm{CN}$ obtained from infrared spectra of ISON on November 19 can be compared to obtain additional insights into the sources of and connection between $\mathrm{HCN}$ and $\mathrm{CN}$ (Fig. $5 \mathrm{C}$ and F).

Based on the spatial distribution of HCN in the coma of ISON on November 19 (Fig. 5C), which is similar to $\mathrm{C}_{2} \mathrm{H}_{6}$ (Fig. 6A) and $\mathrm{H}_{2} \mathrm{O}$ (Fig. 5A), there is no evidence for a significant extended source of HCN. This is in agreement with ALMA observations on November 17.5 that show a symmetric spatial distribution of $\mathrm{HCN}$ suggesting the dominant source of $\mathrm{HCN}$ was released at or close to the nucleus (Cordiner et al., 2014). In contrast to HCN, the spatial distribution of $\mathrm{CN}$ is extended and asymmetric, with a shape broader than dust but with a similar enhancement in the antisolar direction (Fig. 5F). This has important implications for the evolution of $\mathrm{HCN}$ and $\mathrm{CN}$ production in ISON. First, the factor of four or more increase in $\mathrm{HCN} / \mathrm{H}_{2} \mathrm{O}$ mixing ratio between $R_{h}=0.83 \mathrm{AU}$ (November 7 ) and $0.46 \mathrm{AU}$ (November 19) is not due to an increased production of an extended source of $\mathrm{HCN}$ unless the precursor of $\mathrm{HCN}$ has a short lifetime. The increased $\mathrm{HCN} / \mathrm{H}_{2} \mathrm{O}$ mixing ratio is also not obviously associated with the antisolar dust enhancement. This supports the hypothesis that the increasing production of $\mathrm{HCN}$ relative to $\mathrm{H}_{2} \mathrm{O}$ comes from sampling ice layers in the nucleus of different chemistry (perhaps radiation-processed vs. more pristine) rather than the activation of an HCN extended source. Second, the shape of the $\mathrm{CN}$ spatial distribution is consistent with a dominant extended source that is associated with the micron-sized dust seen in the coma and not $\mathrm{HCN}$ or any other $\mathrm{CN}$-bearing volatile (Fig. $5 \mathrm{C}$ and $\mathrm{F}$ ).

In summary, the $\mathrm{HCN} / \mathrm{H}_{2} \mathrm{O}$ mixing ratio appeared to increase by at least a factor of four between $R_{h}=0.83 \mathrm{AU}$ and $0.46 \mathrm{AU}$, with no evidence that this increase is related to the activation of an extended source of $\mathrm{HCN}$ during this time. The predominant source of $\mathrm{CN}$ production is likely a precursor associated with the dust seen at infrared wavelengths with $\mathrm{HCN}$ only a minor contributor to the evolution of overall $\mathrm{CN}$ production and its increase as ISON approached perihelion.

\subsection{9. $H N C$}

HNC, an isomer of $\mathrm{HCN}$, has been detected in several comets at radio wavelengths (e.g. Irvine et al., 1996, 1998; Biver et al., 2002; Lis et al., 2008). HNC has a strong band near $5.0 \mu \mathrm{m}$ but it has not been detected to date at infrared wavelengths. There is strong evidence that the ratio of $\mathrm{HNC} / \mathrm{HCN}$ increases with decreasing heliocentric distance, suggesting a primary source of HNC may be desorption from heated grains (Biver et al., 2002; Irvine et al., 2003). We obtained spectra from a CSHELL setting that targeted both the P6 and P7 lines of HNC and $\mathrm{H}_{2} \mathrm{O}$ on November 19.9 (Tables 1 and 3; Fig. 1F). A $3 \sigma$ upper limit to the HNC production rate was obtained of $Q_{\mathrm{HNC}}<4.4 \times 10^{26}$ molecules s ${ }^{-1}$ with mixing ratios with respect to $\mathrm{H}_{2} \mathrm{O}$ and $\mathrm{HCN}$ of $\mathrm{HNC} / \mathrm{H}_{2} \mathrm{O}<0.13 \%$ and $\mathrm{HNC} / \mathrm{HCN}<65 \%$. Note that $\mathrm{HCN}$ and $\mathrm{HNC}$ production rates were determined $4 \mathrm{~h}$ apart on November 19 as $\mathrm{H}_{2} \mathrm{O}$ production was increasing significantly, so a value of $\mathrm{HNC} / \mathrm{HCN}<50 \%$ is obtained when comparing mixing ratios of these species with respect to $\mathrm{H}_{2} \mathrm{O}$ (Table 4, Fig. 7). The upper limit for HNC derived in this work is in agreement with values obtained on November $15.4\left(R_{h}=0.61 \mathrm{AU}\right)$ of $Q_{\mathrm{HNC}}=6.4 \times 10^{25}$ molecules $\mathrm{s}^{-1}$ and $\mathrm{HNC} / \mathrm{HCN}=18 \%$ (Agúndez et al., 2014), and November $17.5\left(R_{h}=0.54 \mathrm{AU}\right)$ of $Q_{\mathrm{HNC}}=1.2$ $\times 10^{26}$ molecules s $^{-1}$ and $\mathrm{HNC} / \mathrm{HCN}=34 \%$ (Cordiner et al., 2014).

\subsubsection{0. $\mathrm{NH}_{3}$}

$\mathrm{NH}_{3}$ is typically difficult to detect at infrared wavelengths because of a scarcity of strong lines in regions of good atmospheric transmittance. $\mathrm{NH}_{3}$ was targeted on three dates. Two unresolved pairs of $\mathrm{NH}_{3} \mathrm{v}_{1}$ sqR lines were targeted but not detected with NIRSPEC on October 28 , consistent with a $3 \sigma$ upper limit to the production rate $Q_{\mathrm{NH} 3}<1.8 \times 10^{26}$ molecules s${ }^{-1}$ and $\mathrm{NH}_{3} / \mathrm{H}_{2} \mathrm{O}<1.6 \%$ (Tables 2 and 4). On November 19.8, a strong emission from the unresolved $\mathrm{sqR}(1,0)$ and $(1,1)$ line pair was detected giving a production rate of $Q_{\mathrm{NH} 3}=(1.3 \pm 0.3) \times 10^{28}$ molecules s $^{-1}$ and $\mathrm{NH}_{3} / \mathrm{H}_{2} \mathrm{O}=(5.0 \pm 1.2) \%$, which is the highest mixing ratio of $\mathrm{NH}_{3} / \mathrm{H}_{2} \mathrm{O}$ measured in any comet to date. A tentative $(4.7 \sigma)$ detection on November 20.9 is consistent with the value obtained on November 19.8 (Table 4, Fig. 8). We note that $\mathrm{NH}_{3} / \mathrm{HCN}=19 \pm 5$ on November 19 is the highest $\mathrm{NH}_{3} / \mathrm{HCN}$ ratio measured in a comet, rivaled only by the $\mathrm{HCN}$-depleted $6 \mathrm{P} / \mathrm{d}$ 'Arrest with $\mathrm{NH}_{3}$ / $\mathrm{HCN}=16 \pm 6$ (Dello Russo et al., 2009a), and as compared to other measured values at infrared wavelengths which range from $\mathrm{NH}_{3}$ / HCN 0.4 to 7 (Bonev et al., 2009; Dello Russo et al., 2007, 2008 , 2009b, 2011, 2014; DiSanti et al., 2014; Kawakita and Mumma, 2011; Paganini et al., 2012, 2014, 2015; Villanueva et al., 2011b). This is also suggestive of an increase in $\mathrm{NH}_{3} / \mathrm{H}_{2} \mathrm{O}$ in ISON between $R_{h}=1.08 \mathrm{AU}$ (October 28) and 0.46 AU (November 19) (Fig. 8).

We note that any interpretation of $\mathrm{NH}_{3}$ production rates on November 19 and 20 is based on the detection of a single emission; therefore, we cannot entirely rule out that this emission is contaminated by an unknown species. This appears more plausible given the apparent disparity between derived $\mathrm{NH}_{3}$ and $\mathrm{NH}_{2}$ production rates (see Section 3.3.10). We also note that the derived $\mathrm{NH}_{3}$ 
production rate is highly dependent on the assumed rotational temperature. For example, if the rotational temperature of $\mathrm{NH}_{3}$ on November 19 and 20 is $80 \mathrm{~K}$ (as it is for $\mathrm{NH}_{2}$ ) rather than the adopted values of 116 and $136 \mathrm{~K}$, the derived production rates and mixing ratios for $\mathrm{NH}_{3}$ on November 19 and 20 decrease by $\sim 30-40 \%$ to $Q_{\mathrm{NH} 3}=(9.4 \pm 1.8)$ and $(8.7 \pm 3.5) \times 10^{27}$ molecules s $^{-1}$ and $\mathrm{NH}_{3} / \mathrm{H}_{2} \mathrm{O}=(3.6 \pm 0.7) \%$ and $(2.4 \pm 1.0) \%$ respectively. A lower rotational temperature for $\mathrm{NH}_{3}$ compared to $\mathrm{H}_{2} \mathrm{O}$ is feasible as radiative cooling of $\mathrm{NH}_{3}$ from its strong rotational transitions in the far infrared may result in a lower rotational temperature than the surrounding kinetic gas temperature if collisions are not efficient enough to equilibrate the $\mathrm{NH}_{3}$ rotational temperature with that of $\mathrm{H}_{2} \mathrm{O}$, the dominant coma gas.

Because $\mathrm{NH}_{3}$ is difficult to detect at infrared wavelengths, there is no previous information on the spatial distribution for $\mathrm{NH}_{3}$ from infrared measurements of other comets; however, the $\mathrm{NH}_{3}$ emission was sufficiently strong in ISON on November 19 to determine the spatial distribution along the slit (Fig. 5B). The lifetime of $\mathrm{NH}_{3}$ in the solar radiation field at $R_{h}=0.46 \mathrm{AU}$ is $\sim 1200 \mathrm{~s}$ (Huebner et al., 1992), only slightly longer than the lifetime of $\mathrm{H}_{2} \mathrm{CO}$ and shorter than the time it would take a molecule of $\mathrm{NH}_{3}$ to traverse the projected spatial field-of-view along the CSHELL slit. Therefore, if the dominant source of $\mathrm{NH}_{3}$ in the coma is direct release from the nucleus as a parent ice, we expect its spatial distribution to be similar to that of $\mathrm{H}_{2} \mathrm{CO}$ - symmetric and slightly narrower than the spatial distributions for $\mathrm{H}_{2} \mathrm{O}$ and $\mathrm{C}_{2} \mathrm{H}_{6}$. Although the $\mathrm{NH}_{3}$ spatial distribution is symmetric, it instead appears broader and more extended than the spatial distributions of $\mathrm{H}_{2} \mathrm{O}$ and $\mathrm{C}_{2} \mathrm{H}_{6}$ (Fig. 5B), with a multiplicative growth factor more than twice as large as that measured for $\mathrm{H}_{2} \mathrm{O}$ and $\mathrm{OH}$ lines within the same setting (Table 3). The measured column density of molecules within a nucleus-centered $\left(1^{\prime \prime} \times 1^{\prime \prime}\right)$ extract suggests $\mathrm{NH}_{3} / \mathrm{H}_{2} \mathrm{O} \sim 2 \%$, implying that a significant fraction of $\mathrm{NH}_{3}$ is released in the coma as an extended source. However, unlike $\mathrm{CN}$, which is asymmetric and shows enhancement similar to dust, the extension in $\mathrm{NH}_{3}$ is unassociated with any enhancement in the dust spatial distribution in the antisolar direction (Fig. 5B). This suggests that any extended source of $\mathrm{NH}_{3}$ comes from a progenitor volatile ice or from a dust source that is unassociated with micron-sized dust particles seen at infrared wavelengths. It is likely that the apparent increase in $\mathrm{NH}_{3} / \mathrm{H}_{2} \mathrm{O}$ as ISON approached perihelion is due to the activation of an extended source of $\mathrm{NH}_{3}$ prior to $R_{h}=0.46 \mathrm{AU}$. Derivation of a parent scale length from the $\mathrm{NH}_{3}$ spatial distribution is beyond the scope of the current work.

One explanation of the extended $\mathrm{NH}_{3}$ spatial distribution is the recycling of $\mathrm{NH}_{3}$ via ionization and electron recombination reactions in the inner coma such as: $\mathrm{NH}_{3}+\mathrm{H}_{3} \mathrm{O}^{+} \rightarrow \mathrm{NH}_{4}^{+}+\mathrm{H}_{2} \mathrm{O}\left(\mathrm{NH}_{3}\right.$ has a stronger proton affinity than $\mathrm{H}_{2} \mathrm{O}$ ), followed by $\mathrm{NH}_{4}^{+}+\mathrm{e}^{-} \rightarrow$ $\mathrm{NH}_{3}+\mathrm{H}$. It is uncertain whether there is sufficient $\mathrm{H}_{3} \mathrm{O}^{+}$close to the nucleus to make this a viable mechanism for a large extended source of $\mathrm{NH}_{3}$; however, gas and electron densities were increasing rapidly on November 19 and 20 as ISON approached perihelion. Furthermore, it is possible that any $\mathrm{NH}_{3}$ reformed by this mechanism does not easily equilibrate with coma gases by collisions farther from the nucleus and thus will have a lower rotational temperature than the surrounding $\mathrm{H}_{2} \mathrm{O}$ gas.

\subsubsection{1. $\mathrm{NH}_{2}$}

Historically, IR studies of comets have emphasized the parent volatiles. However, $\mathrm{NH}_{2}$ emissions are generally stronger, more numerous, and easier to detect than emissions from its presumptive parent $\mathrm{NH}_{3}$. The ability to measure production rates in both species, when possible, provides a direct test of the importance of $\mathrm{NH}_{3}$ as a parent of $\mathrm{NH}_{2}$. Furthermore, it allows evaluation of the potential of $\mathrm{NH}_{2}$ emissions to be routinely used to indirectly determine $\mathrm{NH}_{3}$ abundances. Recently, a fluorescence model was developed for $\mathrm{NH}_{2}$ IR emissions and applied to Comet C/2004 Q2 (Machholz) (Kawakita and Mumma, 2011). Abundances determined for $\mathrm{NH}_{2}$ and $\mathrm{NH}_{3}$ in $\mathrm{C} / 2004$ Q2 were consistent with $\mathrm{NH}_{3}$ as the dominant parent of $\mathrm{NH}_{2}$; however, this does not appear to be the case for all comets (Dello Russo et al., 2009a).

$\mathrm{NH}_{2}$ emissions were detected on both October 26 (3.6 $\sigma$ detection) and October 28 (6.3 $\sigma$ detection) with derived mixing ratios $\mathrm{NH}_{2} / \mathrm{H}_{2} \mathrm{O}=(0.7 \pm 0.4) \%$ and $(0.4 \pm 0.2) \%$ respectively (Table 4$)$. The relatively high uncertainties in these values stem from the sensitivities of the $\mathrm{NH}_{2} g$-factors to the adopted rotational temperature and the assumption of spherically symmetric and uniform outflow from the nucleus, which is likely not the case for a daughter species such as $\mathrm{NH}_{2}$. These values are in agreement with derived $\mathrm{NH}_{2} / \mathrm{H}_{2} \mathrm{O}$ mixing ratios of $\sim 0.3 \%$ derived from optical measurements on October 25 (McKay et al., 2014). These values are also formally consistent with our derived upper limit for $\mathrm{NH}_{3} / \mathrm{H}_{2} \mathrm{O}<1.6 \%$ on October 28 (Table 4). Measurements of $\mathrm{NH}_{2}$ on November 19.8 and 20.8 show $\mathrm{NH}_{2} / \mathrm{H}_{2} \mathrm{O}=(1.0 \pm 0.2) \%$ and $(0.7 \pm 0.3) \%$ respectively (Table 4$)$, which are not significantly different within the large uncertainties from the October measurements. Interestingly, optical results show no significant increase in $\mathrm{NH}_{2} / \mathrm{H}_{2} \mathrm{O}$ between October $25\left(R_{h}=1.16 \mathrm{AU}\right)$ and November $15\left(R_{h}=0.62 \mathrm{AU}\right)$, contrary to significant increases measured for $\mathrm{CN}$ and $\mathrm{C}_{2}$ during this time period (McKay et al., 2014).

The $\mathrm{NH}_{2}$ production rates and mixing ratios derived on November 19 and 20 are about a factor of five lower than those determined for $\mathrm{NH}_{3}$ on these dates (Table 4). $\mathrm{NH}_{3}$ has a short lifetime in the solar radiation field at these heliocentric distances, and it is expected that $\mathrm{NH}_{3}$ will produce $\mathrm{NH}_{2}$ upon photodissociation with close to $100 \%$ efficiency, so the source of this discrepancy in mixing ratios is unclear and likely indicates an error in the derived infrared $\mathrm{NH}_{2}$ and/or $\mathrm{NH}_{3}$ production rates. We note that part of the discrepancy could be due to the treatment of $\mathrm{NH}_{2}$ as a parent volatile, which likely underestimates the total $\mathrm{NH}_{2}$ production rate; however, it seems unlikely that this by itself accounts for the factor of five difference in derived $\mathrm{NH}_{2}$ and $\mathrm{NH}_{3}$ production rates, and also does not explain discrepancies in production rates for $\mathrm{NH}_{3}$ derived here and $\mathrm{NH}_{2}$ derived at optical wavelengths. A more complete treatment of $\mathrm{NH}_{2}$ production in ISON is beyond the present scope of this paper and is deferred to future work.

The few studies of $\mathrm{NH}_{2}$ and $\mathrm{NH}_{3}$ in comets illustrate the gaps in our understanding of the relationship between these species. Daughter species such as $\mathrm{NH}_{2}$ may be produced by some combination of fluorescence emission and prompt emission. The spatial distribution of a species in the coma can provide information on its release mechanism - solar-pumped IR fluorescence emission of a daughter species will produce a flatter spatial distribution in the coma whereas prompt emission due to dissociative excitation of the parent will follow the parent distribution. The presence of high-excitation $\mathrm{OH}$ lines in IR spectra is an example of a prompt emission mechanism in comets (Bonev et al., 2006). Spatial distributions for $\mathrm{NH}_{2}$ lines seen in IR spectra of Comet C/2004 Q2 (Machholz) show a flatter spatial distribution in the coma than typical parent volatiles (e.g. $\mathrm{H}_{2} \mathrm{O}$ ), and this evidence was used to suggest that $\mathrm{NH}_{2}$ is produced primarily from fluorescence emission (Kawakita and Mumma, 2011). However, because $\mathrm{NH}_{3}$ emissions were too weak to obtain spatial distributions, the full relationship between $\mathrm{NH}_{3}$ and $\mathrm{NH}_{2}$ in that comet is unknown.

In contrast to $\mathrm{C} / 2004 \mathrm{Q} 2$ Machholz, both the $\mathrm{NH}_{3}$ and $\mathrm{NH}_{2}$ emissions were sufficiently strong in ISON on November 19 to determine the spatial distribution along the slit (Fig. 5B and E). The $\mathrm{NH}_{2}$ spatial distribution appears symmetric and extended, similar to the $\mathrm{NH}_{2}$ spatial distribution seen in C/2004 Q2 (Machholz) (Kawakita and Mumma, 2011), and which, taken alone, is consistent with a fluorescence emission mechanism. In our ISON spectra, 
few $\mathrm{NH}_{2}$ lines were covered, and relative intensities on November 19 and 20 are consistent with a fluorescence emission mechanism with a rotational temperature of $\sim 80 \mathrm{~K}$ (Fig. 4). Observations of additional $\mathrm{NH}_{2}$ lines with high lower-state energies, such as seen for $\mathrm{OH}$ in infrared spectra, would provide a stringent test of the emission mechanism. Unfortunately, the limited spectral coverage of CSHELL prohibited the sampling of a sufficient number of $\mathrm{NH}_{2}$ lines to further test the fluorescence versus prompt emission question for ISON.

Within uncertainties the spatial distributions appear similar for $\mathrm{NH}_{2}$ and $\mathrm{NH}_{3}$ inside about $10^{3} \mathrm{~km}$ of the nucleus, with $\mathrm{NH}_{2}$ having a slightly flatter distribution than $\mathrm{NH}_{3}$ outside $10^{3} \mathrm{~km}$ (Fig. 5). Both $\mathrm{NH}_{2}$ and $\mathrm{NH}_{3}$ have flatter spatial distributions than $\mathrm{H}_{2} \mathrm{O}$ and $\mathrm{C}_{2} \mathrm{H}_{6}$ inside about $10^{3} \mathrm{~km}$ of the nucleus (Figs. 5 and 6 ). This is perhaps more consistent with a prompt emission mechanism as a broader $\mathrm{NH}_{2}$ spatial distribution might be expected inside $10^{3} \mathrm{~km}$ if the predominant mechanism were fluorescence emission of $\mathrm{NH}_{2}$ after formation from $\mathrm{NH}_{3}$ in ISON on November 19. As $g$-factors for $\mathrm{NH}_{2}$ lines derived from prompt emission are unknown, values reported here were determined assuming a fluorescence emission mechanism, which could be an additional source of uncertainty in $\mathrm{NH}_{2}$ production rates in ISON on November 19 and 20 if a prompt emission mechanism contributes significantly.

Unlike CN, which shows asymmetric extension in the dustenhanced antisolar direction, $\mathrm{NH}_{2}$ emission is symmetric along the slit and shows no preferential antisolar extension. This is consistent with $\mathrm{NH}_{3}$ or another volatile and not a species associated with the micron-sized dust as its dominant source. It is important to note that because of the unusually high $\mathrm{NH}_{3}$ abundance and the relatively small heliocentric distance of the measurements that the relationship between $\mathrm{NH}_{2}$ and $\mathrm{NH}_{3}$ seen on November 19 in ISON may not be representative of the relationship of these species in other comets. It is possible that for comets with moderate $\mathrm{NH}_{3}$ production rates, such as C/2004 Q2 (Machholz) (Kawakita and Mumma, 2011), a fluorescence emission mechanism may dominate, whereas a prompt emission mechanism could become increasingly important in comets such as ISON that are more enriched in $\mathrm{NH}_{3}$.

\section{Discussion \& summary}

High-resolution infrared spectra of Comet ISON were obtained on four pre-perihelion dates. The production rates and relative abundances of volatiles were directly measured through detected molecular line emissions (Figs. 1 and 2). Spatial distributions for molecular species in the coma were also determined providing information about how these molecules were released from the nucleus and the presence of additional extended sources (Figs. 5 and 6). Comparison of the spatial distributions for related species such as $\mathrm{HCN}, \mathrm{CN}, \mathrm{NH}_{3}$ and $\mathrm{NH}_{2}$ may provide indirect information about the composition of the more volatile dust components. The main conclusions based on derived production rates, spatial distributions, and rotational temperatures determined from infrared spectroscopy of $\mathrm{C} / 2012$ S1 (ISON) are as follows.

(1) The total gas production rate in ISON increased by about a factor of 40 between October $26\left(R_{h}=1.12 \mathrm{AU}\right)$ and November $20\left(R_{h}=0.43 \mathrm{AU}\right)$. Other measurements obtained at this time support this increase and suggest that comet productivity was relatively constant between about $R_{h} \sim 1.3$ and $0.68 \mathrm{AU}$ with a steep increase in productivity inside $R_{h} \sim 0.68 \quad$ (Fig. 7A). Measurements obtained within $R_{h} \sim 0.68$ AU suggest that this increase was not steady but characterized by significant short-term variability (Fig. 7).
(2) There is evidence for a significant increase in $\mathrm{H}_{2} \mathrm{O}$ production rate on November 19 from the beginning to the end of our observing block (17:00-23:10). Within uncertainties $\mathrm{H}_{2} \mathrm{O}$ production remained stable at $\sim 2.3-2.8 \times 10^{29}$ molecules $\mathrm{s}^{-1}$ between 17:00 and 19:45 with a steady increase beginning shortly thereafter until the final measurement of $(4.4 \pm 0.3) \times 10^{29}$ molecules $\mathrm{s}^{-1}$ at 23:06 (Fig. 7B). Three measurements of the $\mathrm{H}_{2} \mathrm{O}$ production rate on November 20 are consistent with the values derived in the last two grating settings obtained on November 19 (Fig. 7B).

(3) There is evidence that the relative chemical abundances of some species changed in ISON as it approached perihelion. These compositional changes may partly reflect a transition from sampling radiation-processed outer layers to sampling gradually more pristine natal material. Unsaturated species such as $\mathrm{HCN}, \mathrm{C}_{2} \mathrm{H}_{2}$, and $\mathrm{H}_{2} \mathrm{CO}$, that are readily depleted by hydrogen atom addition reactions or other chemical reactions in the nucleus ices, showed increasing mixing ratios with respect to saturated species such as $\mathrm{H}_{2} \mathrm{O}, \mathrm{C}_{2} \mathrm{H}_{6}$ and $\mathrm{CH}_{3} \mathrm{OH}$ as ISON approached the Sun. It is interesting to note that $\mathrm{C}_{2} \mathrm{H}_{2} / \mathrm{C}_{2} \mathrm{H}_{6}$ measured in ISON on November 19 is similar to the ratio in another dynamically new comet, C/2006 P1 (McNaught), also measured at a small $R_{h}$ (Dello Russo et al., 2009b). Alternatively, the abundances of some species on November 19 and 20 (e.g. $\mathrm{NH}_{3}, \mathrm{OCS}$, and $\mathrm{H}_{2} \mathrm{CO}$ ) may be enhanced by the thermal activation of extended sources from volatiles or grains not associated with the micronsized dust source seen at infrared wavelengths as ISON approached perihelion. However, our observations lack the temporal coverage to allow a detailed determination of how the relative abundances of all measured species evolved as ISON approached perihelion.

(4) Compared to mean values in comets measured to date at infrared wavelengths, the abundances of species with respect to $\mathrm{H}_{2} \mathrm{O}$ are classified as follows: $\mathrm{CH}_{4}$ (depleted at $R_{h}=1.1 \mathrm{AU}$ ), $\mathrm{C}_{2} \mathrm{H}_{6}$ (depleted), $\mathrm{CH}_{3} \mathrm{OH}$ (depleted), $\mathrm{C}_{2} \mathrm{H}_{2}$ (typical or depleted at $R_{h}=1.1 \mathrm{AU}$, slightly enhanced by $R_{h}=0.46 \mathrm{AU}$ ), HCN (depleted for $R_{h} \geqslant 0.83 \mathrm{AU}$ (DiSanti et al., in press), typical by $R_{h}=0.46 \mathrm{AU}$ ), $\mathrm{NH}_{3}$ (upper limits in the typical range for $R_{h}=1.1 \mathrm{AU}$, strongly enhanced by $R_{h}=0.46 \mathrm{AU}$ ), $\mathrm{H}_{2} \mathrm{CO}$ (in typical range for $R_{h} \geqslant 0.83 \mathrm{AU}$ (DiSanti et al., in press), enhanced at $R_{h}=0.46 \mathrm{AU}$ ), OCS (typical at $\left.R_{h}=0.46 \mathrm{AU}\right) . \mathrm{NH}_{3} / \mathrm{HCN}, \mathrm{H}_{2} \mathrm{CO} / \mathrm{CH}_{3} \mathrm{OH}$, and $\mathrm{C}_{2} \mathrm{H}_{2} / \mathrm{C}_{2} \mathrm{H}_{6}$ measured on November 19 at $R_{h}=0.46 \mathrm{AU}$ are among the highest ratios measured in comets (Fig. 8).

(5) Spatially resolved column abundances (spatial distributions) for $\mathrm{H}_{2} \mathrm{O}, \mathrm{C}_{2} \mathrm{H}_{6}, \mathrm{C}_{2} \mathrm{H}_{2}, \mathrm{HCN}$, and $\mathrm{H}_{2} \mathrm{CO}$ are all symmetric, with peak column densities slightly offset in the sunward direction from the peak of the dust emission, consistent with primary release from ices within or very close to the nucleus. There is no evidence from the spatial distributions alone for large contributions due to extended sources (Figs. 5 and 6). OCS appears symmetric but slightly extended compared to its short lifetime, which may suggest a significant contribution from an extended source (Fig. 6C). The symmetric and $\mathrm{H}_{2} \mathrm{O}$-like spatial distributions for $\mathrm{C}_{2} \mathrm{H}_{2}$ and $\mathrm{HCN}$ suggest that any increase in $\mathrm{C}_{2} \mathrm{H}_{2}$ and $\mathrm{HCN}$ production rates with respect to $\mathrm{H}_{2} \mathrm{O}$ between $\sim 1.1$ and $0.46 \mathrm{AU}$ were not caused by the activation of extended sources (either volatile or dust) as ISON approached the Sun.

(6) The spatial distribution for $\mathrm{NH}_{3}$ is symmetric and extended, which is consistent with a significant contribution from an extended source that is not associated with micron-sized dust extension in the antisolar direction (Fig. 5B). The spatial distribution for $\mathrm{NH}_{2}$ is similar to $\mathrm{NH}_{3}$, consistent with $\mathrm{NH}_{3}$ as the primary parent of $\mathrm{NH}_{2}$ in ISON (Fig. 5E). However, there 
is a significant and unexplained discrepancy between derived $\mathrm{NH}_{3}$ and $\mathrm{NH}_{2}$ production rates with $\mathrm{NH}_{3}$ values about a factor of five higher (Table 4). Possible explanations or contributors to this discrepancy include: (1) A contamination from an unknown species to the single $\mathrm{NH}_{3}$ emission line detected on November 19 and 20 resulting in an erroneously high $\mathrm{NH}_{3}$ production rate. (2) Overestimation of the $\mathrm{NH}_{3}$ production rate due to a few other potential factors (see Section 3.3.9) (3) The $\mathrm{NH}_{2}$ production rates were derived from a classical Haser model and assume fluorescence emission. Because $\mathrm{NH}_{2}$ is a daughter species with possible contributions from a prompt emission mechanism, these derived production rates are highly uncertain.

(7) The spatial distribution for $\mathrm{CN}$ is extended and asymmetric, with a shape broader than dust but with a similar enhancement in the antisolar direction (Fig. 5F). This is notably different from the narrow and symmetric HCN distribution (Fig. 5C). This is evidence that the primary parent of $\mathrm{CN}$ is an extended source associated with the micron-sized dust and not $\mathrm{HCN}$. Comparison of $\mathrm{HCN}$ and $\mathrm{CN}$ production rates from infrared and optical measurements are also consistent with $\mathrm{HCN}$ as only a minor source of $\mathrm{CN}$ in ISON.

(8) Comparison of $\mathrm{C}_{2}, \mathrm{C}_{2} \mathrm{H}_{2}$ and $\mathrm{C}_{2} \mathrm{H}_{6}$ production rates from infrared and optical measurements suggest that $\mathrm{C}_{2} \mathrm{H}_{2}$ and $\mathrm{C}_{2} \mathrm{H}_{6}$ are not the primary parents of $\mathrm{C}_{2}$ in ISON.

(9) The spatially resolved $\mathrm{H}_{2} \mathrm{O}$ rotational temperature on November $19\left(R_{h}=0.46 \mathrm{AU}\right)$ shows an antisolar asymmetry, which may represent an intermediate case between the observations of Bonev et al. (2014) at $R_{h}=0.53$ and $0.35 \mathrm{AU}$ and provides a snapshot within this time of rapid coma evolution (Fig. 3). The comparison of spatially resolved $\mathrm{H}_{2} \mathrm{O}$ column densities and rotational temperatures in ISON presented in this work and in Bonev et al., 2014 provide the most stringent observational constraints to date on the importance of photochemical heating, icy grain release, and adiabatic cooling on gas rotational temperatures in the inner coma of comets at small heliocentric distances.

\section{Acknowledgments}

Data were obtained at the NASA Infrared Telescope Facility operated by the University of Hawaii under cooperative agreement number NNX-08AE38A with the NASA Science Mission Directorate, Planetary Astronomy Program. Data presented herein were also obtained at the W.M. Keck Observatory, which is operated as a scientific partnership among the California Institute of Technology, the University of California and the National Aeronautics and Space Administration. The Observatory was made possible by the generous financial support of the W.M. Keck Foundation. We thank NASA, the Comet ISON Observing Campaign (CIOC), and the IRTF and Keck Observatories for setting aside campaign time for these observations, and the former NASA PAST and PATM Programs for their financial support of this work. MAD thanks the NASA SSW Program, grant NNX15AH29G for support. We note that all raw data from observations presented here are publicly available through IRTF and Keck archives. The authors wish to recognize and acknowledge the very significant cultural role and reverence that the summit of Mauna Kea has always had within the indigenous Hawaiian community. We are most fortunate to have the opportunity to conduct observations from this mountain.

\section{References}

Agúndez, M. et al., 2014. Molecular observations of Comets C/2012 S1 (ISON) and C 2013 R1 (Lovejoy): HNC/HCN ratios and upper limits to $\mathrm{PH}_{3}$. Astron. Astrophys. 564, L2 (5pp.).
A'Hearn, M.F. et al., 1995. The ensemble properties of comets: Results from narrowband photometry of 85 comets, 1976-1992. Icarus $118,223-270$.

Allen, M. et al., 1987. Evidence for methane and ammonia in the coma of comet P/Halley. Astron. Astrophys. 187, 502-512.

Bast, J.E. et al., 2013. Exploring organic chemistry in planet-forming zones. Astron. Astrophys. 551, A118 (24pp.).

Biver, N. et al., 1999. Long term evolution of the outgassing of Comet Hale-Bopp from radio observations. Earth Moon Planets 78, 5-11.

Biver, N. et al., 2002. Chemical composition diversity among 24 comets observed at radio wavelengths. Earth Moon Planets 90, 323-333.

Bockelée-Morvan, D., Crovisier, J., 1992. Formaldehyde in comets II. Excitation of the rotational lines. Astron. Astrophys. 264, 282-291.

Bockelée-Morvan, D. et al., 2000. New molecules found in Comet C/1995 01 (HaleBopp). Investigating the link between cometary and interstellar material. Astron. Astrophys. 353, 1101-1114.

Bockelée-Morvan, D. et al., 2005. The composition of cometary volatiles. In: Festou, M.C., Keller, H.U., Weaver, H.A. (Eds.), Comets II. Univ. of Arizona Press, Tucson, pp. 391-423.

Bodewits, D., Farnham, T. A'Hearn, M.F, 2013 C/2012 S1 (ISON). CBET 3718, 1.

Bonev, B.P., 2005. Towards a Chemical Taxonomy of Comets: Infrared Spectroscopic Methods for Quantitative Measurements of Cometary Water (With an Independent Chapter on Mars Polar Science). Ph.D. Dissertation, Univ. of Toledo.

Bonev, B.P. et al., 2006. A comprehensive study of infrared $\mathrm{OH}$ prompt emission in two comets. I. Observations and effective g-factors. Astrophys. J. 653, 774-787.

Bonev, B.P. et al., 2007. A search for variation in the $\mathrm{H}_{2} \mathrm{O}$ ortho-para ratio and rotational temperature in the inner coma of Comet C/2004 Q2 (Machholz). Astrophys. J. 661, L97-L100.

Bonev, B.P. et al., 2008a. IRCS/Subaru observations of water in the inner coma of Comet 73P/Schwassmann-Wachmann 3: Spatially resolved rotational temperatures and ortho para ratios. Icarus 196, 241-248.

Bonev, B.P. et al., 2008b. The peculiar volatile composition of Comet 8P/Tuttle: A contact binary of chemically distinct cometesimals? Astrophys. J. 680, L61-L64.

Bonev, B.P. et al., 2009. Comet C/2004 Q2 (Machholz): Parent volatiles, a search for deuterated methane, and constraint on the $\mathrm{CH}_{4}$ spin temperature. Astrophys. J. 699, 1563-1572.

Bonev, B.P. et al., 2013. Evidence for two modes of water release in Comet 103P/ Hartley 2: Distributions of column density, rotational temperature, and orthopara ratio. Icarus 222, 740-751.

Bonev, B.P. et al., 2014. The inner coma of Comet C/2012 S1 (ISON) at 0.53 AU and 0.35 AU from the Sun. Astrophys. J. 796, L6 (6pp.).

Cochran, A.L., Barker, E.S., Gray, C.L., 2012. Thirty tears of cometary spectroscopy from McDonald Observatory. Icarus 218, 144-168.

Colangeli, L. et al., 2005. Laboratory experiments on cometary materials. In: Festou, M.C., Keller, H.U., Weaver, H.A. (Eds.), Comets II. Univ. of Arizona Press, Tucson, pp. 695-717.

Combi, M.R., Delsemme, A.H., 1986. Neutral cometary atmospheres. V - $C_{2}$ and CN in comets. Astrophys. J. 308, 472-484.

Combi, M.R., Fink, U., 1997. A critical study of molecular photodissociation and CHON grain sources for cometary $C_{2}$. Astrophys. J. 484, 879-890.

Combi, M.R. et al., 1999. Observation and analysis of high-resolution optical line profiles in Comet Hyakutake (C/1996 B2). Astrophys. J. 512, 961-968.

Combi, M.R. et al., 2014. Unusual water production activity of Comet C/2012 S1 (ISON): Outbursts and continuous fragmentation. Astrophys. J. 788, L7 (5pp.).

Cordiner, M.A. et al., 2014. Mapping the release of volatiles in the inner comae of Comets C/2012 F6 (Lemmon) and C/2012 S1 (ISON) using the Atacama Large Millimeter/Submillimeter Array. Astrophys. J. 792, L2 (6pp.).

Crovisier, J. et al., 2013. C/2012 S1 (ISON). CBET 3711, 1.

Dello Russo, N. et al., 1998. Carbonyl sulfide in Comets C/1996 B2 (Hyakutake) and C/1995 01 (Hale-Bopp): Evidence for an extended source in Hale-Bopp. Icarus 135, 377-388.

Dello Russo, N. et al., 2001. Ethane production and release in Comet C/1995 01 Hale-Bopp. Icarus 153, 162-179.

Dello Russo, N. et al., 2002. Production of $\mathrm{C}_{2} \mathrm{H}_{6}$ and $\mathrm{H}_{2} \mathrm{O}$ in Comet $\mathrm{C} 1$ Ikeya-Zhang on one date. In: Warmbein, B. (Ed.), The Proceedings for the Asteroids, Comets, Meteors Conference, Berlin, Germany 2002. ESA SP, vol. 500. ESA, Nordwijk, pp. 689-692.

Dello Russo, N. et al., 2004. Water production and release in Comet 153P/IkeyaZhang (C/2002 C1): Accurate rotational temperature retrievals from hot-band lines near $2.9-\mu \mathrm{m}$. Icarus 168, 186-200.

Dello Russo, N. et al., 2005. Water production rates, rotational temperatures, and spin temperatures in Comets C/1999 H1 (Lee), C/1999 S4, and C/2001 A2. Astrophys. J. 621, 537-544.

Dello Russo, N. et al., 2006. A high-resolution infrared spectral survey of Comet C/ 1999 H1 Lee. Icarus 184, 255-276.

Dello Russo, N. et al., 2007. Compositional homogeneity in the fragmented Comet 73P/Schwassmann-Wachmann 3. Nature 448, 172-175.

Dello Russo, N. et al., 2008. The volatile chemistry of Comet 17P/Holmes after its extraordinary outburst. Astrophys. J. 680, 793-804.

Dello Russo, N. et al., 2009a. The parent volatile composition of 6P/d'Arrest and a chemical comparison of Jupiter-family comets measured at infrared wavelengths. Astrophys. J. 703, 187-197.

Dello Russo, N. et al., 2009b. Infrared measurements of the chemical composition of C/2006 P1 McNaught. Icarus 200, 271-279.

Dello Russo, N. et al., 2011. The volatile composition and activity of Comet 103P/ Hartley 2 during the EPOXI closest approach. Astrophys. J. 734, L8 (6pp.). 
Dello Russo, N. et al., 2014. The volatile composition of 81P/Wild 2 from groundbased high-resolution infrared spectroscopy. Icarus 238, 125-136.

DiSanti, M.A. et al., 2001. Carbon monoxide production and excitation in Comet C/1995 01 (Hale-Bopp): Isolation of native and distributed CO sources. Icarus 153, 361-390.

DiSanti, M.A. et al., 2002. $\mathrm{CO}, \mathrm{H}_{2} \mathrm{CO}$, and $\mathrm{CH}_{3} \mathrm{OH}$ in Comet $\mathrm{C} / 2002 \mathrm{C} 1$ Ikeya-Zhang. In: Warmbein, B. (Ed.), The Proceedings for the Asteroids, Comets, Meteors Conference, Berlin, Germany 2002. ESA SP, vol. 500. ESA, Nordwijk, pp. 571574

DiSanti, M.A. et al., 2006. Detection of formaldehyde emission in Comet C/2002 T7 (LINEAR) at infrared wavelengths: Line-by-line validation of modeled fluorescent intensities. Astrophys. J. 650, 470-483.

DiSanti, M.A. et al., 2009. A multi-wavelength study of parent volatile abundances in Comet C/2006 M4 (SWAN). Icarus 203, 589-598.

DiSanti, M.A. et al., 2013. Highly depleted ethane and mildly depleted methanol in Comet 21P/Giacobini-Zinner: Application of a new empirical $v_{2}$-band model for $\mathrm{CH}_{3} \mathrm{OH}$ near 50 K. Astrophys. J. 763, 1 (19pp.).

DiSanti, M.A. et al., 2014. Pre- and post-perihelion observations of C/2009 P1 (Garradd): Evidence for an oxygen-rich heritage? Icarus 228, 167-180.

DiSanti, M.A. et al., 2015. En route to destruction: The evolution in composition of ices in C/2012 S1 (ISON) between 1.2 and $0.34 \mathrm{AU}$ from the Sun as revealed at infrared wavelengths. Astrophys. J., in press.

Fink, U., 2009. A taxonomic survey of comet composition 1985-2004 using CCD spectroscopy. Icarus 201, 311-334.

Fougere, N. et al., 2012. Understanding measured water rotational temperatures and column densities in the very innermost coma of Comet 73P/SchwassmannWachmann 3 B. Icarus 221, 174-185.

Fuchs, G.W. et al., 2009. Hydrogenation reactions in interstellar CO ice analogues: A combined experimental/theoretical approach. Astron. Astrophys. 505, 629-639.

Gibb, E.L. et al., 2003. Methane in Oort cloud comets. Icarus 165, 391-406.

Gibb, E.L. et al., 2012. Chemical composition of Comet C/2007 N3 (Lulin): Another "atypical" comet. Astrophys. J. 750, 102 (14pp.).

Greene, T.P. et al., 1993. CSHELL: A high spectral resolution 1-5 $\mu \mathrm{m}$ cryogenic echelle spectrograph for the IRTF. Proc. SPIE 1946, 313-324.

Helbert, J. et al., 2005. The chemistry of $C_{2}$ and $C_{3}$ in the coma of Comet C/1995 01 (Hale-Bopp) at heliocentric distances $r_{h} \geqslant 2.9$ AU. Astron. Astrophys. 442, 1107-1120.

Hiraoka, K. et al., 2000. Study of the reactions of $\mathrm{H}$ and D atoms with solid $\mathrm{C}_{2} \mathrm{H}_{2}$, $\mathrm{C}_{2} \mathrm{H}_{4}$ and $\mathrm{C}_{2} \mathrm{H}_{6}$ at cryogenic temperatures. Astrophys. J. 532, 1029-1037.

Huebner, W.F., Keady, J.J., Lyon, S.P., 1992. Solar photo rates for planetary atmospheres and atmospheric pollutants. Astrophys. Space Sci. 195, 1-294.

Irvine, W.M. et al., 1996. Spectroscopic evidence for interstellar ices in Comet Hyakutake. Nature 383, 418-420.

Irvine, W.M. et al., 1998. Chemical processing in the coma as the source of cometary HNC. Nature 393, 547-550.

Irvine, W.M. et al., 2003. HCN and HNC in Comets C/2000 Wm1 (LINEAR) and C/ 2002 C1 (Ikeya-Zhang). Oig. Life Evol. Biosph. 33, 609-619.

Jehin, E. et al., 2011. TRAPPIST: TRAnsiting Planets and PlanetesImals Small Telescope. The Messenger 145, 2-6.

Jessberger, E.K., Christoforidis, A., Kissel, J., 1988. Aspects of the major element composition of Halley's dust. Nature 332, 691-695.

Kaiser, R.I., Balucani, N., 2002. Astrobiology - The final frontier in chemical reaction dynamics. Int. J. Astrobiol. 1, 15-23.

Kawakita, H. et al., 2003. Saturated hydrocarbons in Comet 153P/Ikeya-Zhang: Ethane, methane, and monodeuterio-methane. Astrophys. J. 590, 573-578.

Kawakita, H. et al., 2005. Nuclear spin temperature and deuterium-to-hydrogen ratio of methane in Comet C/2001 Q4 (NEAT). Astrophys. J. 623, L49-L52.

Kawakita, H., Mumma, M.J., 2011. Fluorescence excitation models of ammonia and amidogen radical $\left(\mathrm{NH}_{2}\right)$ in comets: Application to Comet C/2004 Q2 (Machholz). Astrophys. J. 727, 91 (11pp.).

Keane, J.V. et al., 2013. C/2013 S1 (ISON). IAU Circ. 9261, 1.

Kobayashi, H. et al., 2010. High-dispersion infrared spectroscopic observations of Comet 8P/Tuttle with VLT/CRIRES. Astron. Astrophys. 509, A80 (9pp.).

Lis, D.C. et al., 2008. Hydrogen isocyanide in Comet 73P/Schwassmann-Wachmann (Fragment B). Astrophys. J. 675, 931-936.

Magee-Sauer, K. et al., 1999. Infrared spectroscopy of the $v_{3}$ band of hydrogen cyanide in C/1995 01 Hale-Bopp. Icarus 142, 498-508.
Magee-Sauer, K. et al., 2002. Production of $\mathrm{HCN}$ and $\mathrm{C}_{2} \mathrm{H}_{2}$ in Comet $\mathrm{C} / 2002 \mathrm{C} 1$ Ikeya-Zhang on UT April 13.8.2002. In: Warmbein, B. (Ed.), The Proceedings for the Asteroids, Comets, Meteors Conference, Berlin, Germany 2002. ESA SP, vol. 500. ESA, Nordwijk, pp. 549-552.

Magee-Sauer, K. Mumma, M.J. DiSanti, M.A, Dello Russo, N Gibb, E.L., Bonev, B.P. Villanueva, G.L., 2008. The organic composition of comet C/2001 A2 (LINEAR) I. Evidence for an unusual organic chemistry. Icarus 194, 347-356.

McKay, A. et al., 2014. Evolution of fragment-species production rates in Comet C 2012 S1 (ISON) from 1.6 AU to 0.4 AU. In: Muinonen, K. et al. (Eds.), Asteroids, Comets, Meteors 2014 Proceedings Held 30 June-4 July, 2004 in Helsinki, Finland.

McLean, I.A. et al., 1998. The design and development of NIRSPEC: A near-infrared echelle spectrograph for the Keck II telescope. Proc. SPIE 3354, 566-578.

Meier, R. et al., 1993. The extended formaldehyde source in Comet P/Halley. Astron. Astrophys. 277, 677-690.

Mumma, M.J. et al., 2001a. Organic composition of C/1999 S4 (LINEAR): A comet formed near Jupiter? Science 292, 1334-1339.

Mumma, M.J. et al., 2001b. A survey of organic volatile species in Comet C/1999 H (Lee) using NIRSPEC at the Keck Observatory. Astrophys. J. 546, 1183-1193.

Mumma, M.J. et al., 2005. Parent volatiles in Comet 9P/Tempel 1: Before and after impact. Science 310, 270-274.

Mumma, M.J., Charnley, S.B., 2011. The chemical composition of comets - Emerging taxonomies and natal heritage. Annu. Rev. Astron. Astrophys. 49, 471-524.

Mumma, M.J. et al., 2011. Temporal and spatial aspects of gas release during the 2010 apparition of Comet 103P/Hartley-2. Astrophys. J. 734, L7 (6pp.).

Mumma, M.J. et al., 2013a. Comet C/2012 S1 (ISON). IAU Circ. 9263, 2.

Mumma, M.J. et al., 2013b. Comet C/2012 S1 (ISON). IAU Circ. 9261, 2

Opitom, C. et al., 2013a. Comet C/2012 S1 (ISON). CBET 3711, 3.

Opitom, C. et al., 2013b. Comet C/2012 S1 (ISON). CBET 3693, 1.

Paganini, L. et al., 2012. The formation heritage of Jupiter family Comet 10P/Tempel 2 as revealed by infrared spectroscopy. Icarus 218, 644-653.

Paganini, L. et al., 2013. Comet C/2012 S1 (ISON). IAU Circ. 9263, 3.

Paganini, L. et al., 2014. C/2013 R1 (Lovejoy) at IR wavelengths and the variability of CO abundances among Oort cloud comets. Astrophys. J. 791, 122 (8pp.).

Paganini, L. et al., 2015. The volatile composition of Comet C/2003 K4 (LINEAR) a near-IR wavelengths - Comparisons with results from the Nancay Radio Telescope and from the Odin, Spitzer, and SOHO Space Observatories. Astrophys. J. 808, 1 (8pp.).

Radeva, Y.L. et al., 2010. The organic composition of Comet C/2000 WM $\mathrm{WM}_{1}$ (LINEAR) revealed through infrared spectroscopy. Icarus 206, 764-777.

Radeva, Y.L. et al., 2013. High-resolution infrared spectroscopic measurements of Comet 2P/Encke: Unusual organic composition and low rotational temperatures. Icarus 223, 298-307.

Rettig, T.W. et al., 1992. Comet outbursts and polymers of HCN. Astrophys. J. 398 293-298.

Theule, P. et al., 2011. Hydrogenation of solid hydrogen cyanide HCN and methanimine $\mathrm{CH}_{2} \mathrm{NH}$ at low temperature. Astron. Astrophys. 534, A64 (6pp.).

Villanueva, G.L., Mumma, M.J., Magee-Sauer, K., 2011a. Ethane in planetary and cometary atmospheres: Transmittance and fluorescence models of the $v_{7}$ band at $3.3 \mu \mathrm{m}$. J. Geophys. Res. 116, E08012. http://dx.doi.org/10.1029/ 2010JE003794.

Villanueva, G.L. et al., 2011b. The molecular composition of Comet C/2007 W1 (Boattini): Evidence of a peculiar outgassing and a rich chemistry. Icarus 216 227-240.

Villanueva, G.L. et al., 2012. A quantum band model of the $v_{3}$ fundamental band of methanol $\left(\mathrm{CH}_{3} \mathrm{OH}\right)$ and its application to fluorescence spectra of comets. Astrophys. J. 747, 37 (11pp.).

Weaver, H. et al., 2013. C/2013 S1 (ISON). CBET 3680, 1.

Weiler, M., 2011. The chemistry of $C_{3}$ and $C_{2}$ in cometary comae. I. Current models revised. Astron. Astrophys. http://dx.doi.org/10.1051/0004-6361/201117480.

Woodney, L.M., McMullin, J., A'Hearn, M.F., 1997. Detection of OCS in Comet Hyakutake (C/1996 B2). Planet. Space Sci. 45, 717-719.

Xie, X., Mumma, M.J., 1996. Monte Carlo simulation of cometary atmospheres: Application to Comet P/Halley at the time of the Giotto spacecraft encounter. Il. Axisymmetric model. Astrophys. J. 464, 457-475. 